The Free Internet Journal

for Organic Chemistry
Paper

Arkivoc 2017, iii, 302-315

\title{
The synthesis of thioglucosides substituted 1,4-naphthoquinones and their conversion in oxathiane fused quinone-thioglucoside conjugates
}

\author{
Yuri E. Sabutskii, Vladimir A. Denisenko, Roman S. Popov, and Sergey G. Polonik*
}

G.B. Elyakov Pacific Institute of Bioorganic Chemistry Far East Branch Russian Academy of Sciences, Prospekt 100-let Vladivostoku 159, Vladivostok 690022, Russia

Email: sergpol@piboc.dvo.ru

Dedicated to Prof. Oleg A. Rakitin on the occasion of his 65th anniversary

Received 07-29-2017

Accepted 08-11-2017

Published on line 08-31-2017

\section{Abstract}

In this paper we describe a methodology for the preparation of thioglucosides of substituted 1,4naphthoquinones via condensation of related chloronaphthoquinones with the sodium salt of 1 -thio- $\beta$-Dglucopyranose in acetone-MeOH solution and subsequent base-catalytic conversion of these mono- and dithioglucosides in the linear tetracyclic quinone-carbohydrate conjugates.<smiles></smiles>

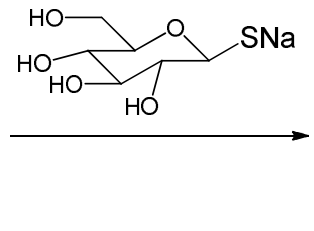<smiles>[R]C1=C([R])C2=C([R])C([R])C(=O)C(=O)C2=C([R])C1=O</smiles><smiles>[R]c1c([R])c([R])c2c(c1[R])C(=O)C1=C(S[C@@H]3O[C@@H](CO)[C@@H](O)[C@H](O)[C@@H]3O1)C2=O</smiles>

$\mathrm{R}=\mathrm{Cl}, \mathrm{OMe}, \mathrm{SG} ; \mathrm{R}^{1}=\mathrm{H}, \mathrm{OH}, \mathrm{OMe} ; \mathrm{R}^{2}=\mathrm{H}, \mathrm{Me}, \mathrm{Cl} ; \mathrm{SG}=1$-thioglucopyranosyl

Keywords: 1-Thioglucose sodium salt, 1,4-naphthoquinones, thioglucosides, heterocyclisation. 


\section{Introduction}

The 1,4-napthoquinones are widely distributed in nature and occur in animals, plants and microorganisms ${ }^{1}$. This group of compounds exhibits antibacterial, ${ }^{2}$ cardioprotective ${ }^{3}$ and anticancer activities ${ }^{4-5}$ and provide structures regarded as perspectives in medicinal chemistry. Quinones exert their actions through the generation of reactive oxygen species and modulation of redox signaling radical reactions: as prooxidants; as antioxidants and as electrophiles, forming covalent bonds with tissue nucleophiles. ${ }^{6}$ That broad spectrum of biological activity renders them interesting leads for the development of novel medicines. ${ }^{7}$ Naphthoquinones often possess poor solubility which hampered their practical use. The conjugation of naphthoquinones with non-toxic carbohydrates is one of the best successful way for improving their solubility. Also, conjugation of naphthoquinones with carbohydrates led to the novel structures with new types of biological activity. ${ }^{8-11}$

In the course of our drug research project we developed an effective method for preparation of naphthoquinone acetylthioglucosides by the condensation of available substituted chloroquinones $\mathbf{1 a}, \mathbf{b}$ with tetra-O-acetyl-1-thio- $\beta$-D-glucopyranose (2) (AGSH) and obtained related naphthoquinone acetylglucosides $\mathbf{3 a}, \mathbf{b}$. The acetylglucoside naphthoquinones $\mathbf{3} \mathbf{a}, \mathbf{b}$ readily were deacetylated with $\mathrm{MeONa} / \mathrm{MeOH}$ and led the water soluble thioglucosides $\mathbf{4 a} \mathbf{a}, \mathbf{b}$. However under these base conditions thioglucosides $\mathbf{4 a , b}$ immediately converted in the insoluble quinone-sugar tetracyclic conjugate $\mathbf{5 a}$ in good yield ${ }^{12}$ (Scheme 1). Recently, this protocol was successfully used to prepare quinone-sugar tetracyclic conjugates based on quinone $\mathbf{1 b}$ with acetylated 1-mercaptosugars of D-galactose, D-mannose, D-xylose, L-arabinose and D-maltose. ${ }^{13}$ The obtained sugar-quinone tetracycles were converted in acetyl derivatives by the treatment $\mathrm{Ac}_{2} \mathrm{O} / \mathrm{Py}$. Both synthesized tetracyclic quinone conjugates and their acetylated tetracylic derivatives were active in vitro against human promyelocytic leukemia $\mathrm{HL}-60$ in 1.0-5.0 $\mu \mathrm{M}$ concentrations, while starting acyclic acetylglycosides were less active $~ 10-100$ times. $^{14}$

Previous work:

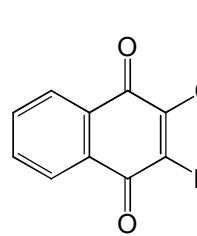

1a: $\mathrm{R}=\mathrm{Cl}$ 1b: $R=O M e$

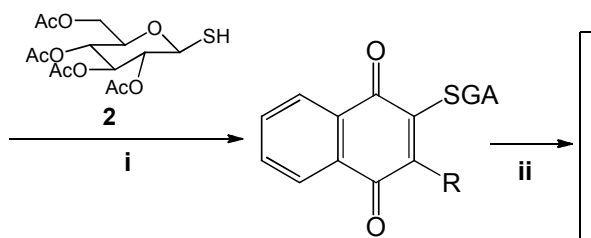

3a: $R=S G A$ 3b: $R=O M e$

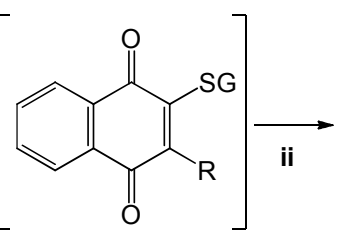

4a: $R=S G$ 4b: $\mathrm{R}=\mathrm{OMe}$

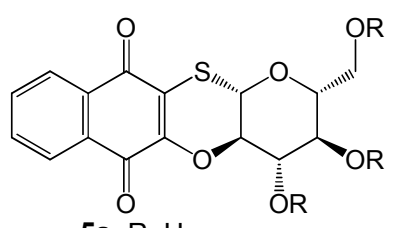

5a: $\mathrm{R}=\mathrm{H} \quad \longrightarrow$ iii

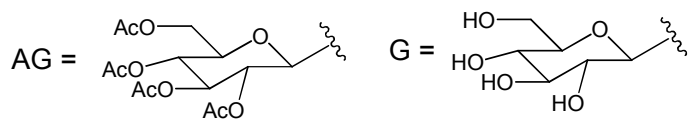

Scheme 1. Reagents and conditions: (i) $\mathrm{K}_{2} \mathrm{CO}_{3}$, acetone, r,t.; (ii) $\mathrm{MeONa} / \mathrm{MeOH}$, r.t.; (iii) $\mathrm{Ac}{ }_{2} \mathrm{O} / \mathrm{Py}$, rt.

\section{Results and Discussion}

In present work we describe a methodology for the preparation of base-sensitive thioglucosides of various 1,4naphthoquinones by the condensation of substituted chloronapthoquinones with the sodium salt of 1-thio- $\beta$ D-glucopyranose (6) and a base-catalytic conversion of these thioglucosides to tetracyclic quinonecarbohydrate conjugates. The thioglucose sodium salt $\mathbf{6}$ is the good nucleophile and a weak base, therefore it 
can be used for direct preparation of naphthoquinone thioglucosides without base-catalyzed deacetylation procedure.

Present work:

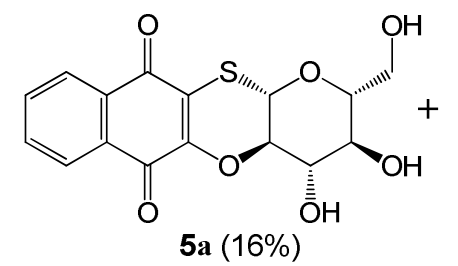

$5 \mathbf{a}(16 \%)$

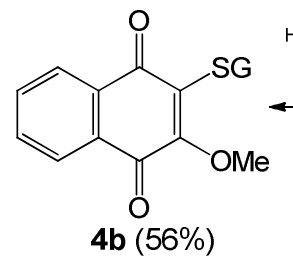

4b $(56 \%)$

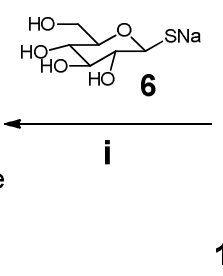

1a R=Cl, 1b R=OMe

$S G=1$-thioglucopyranosyl

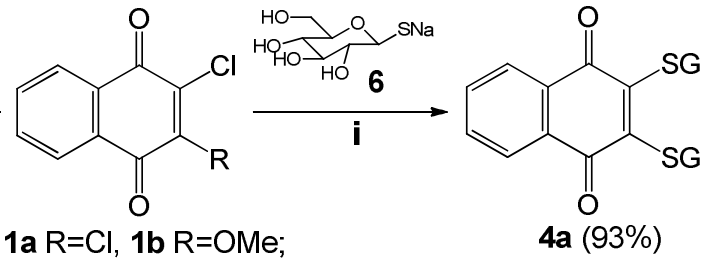

$4 a(93 \%)$

Scheme 2. Reagents and conditions: (i) acetone/MeOH, rt.;

The study was performed on substituted 2,3-dichloro- and 2-methoxy-3-chloro-1,4-naphthoquinones, which were converted in naphthoquinone mono- and dithioglucosides (Schemes 2, 4 and 6). Initially, we examined the utility of 1-thioglucose $\mathbf{6}$ on model compounds $\mathbf{1} \mathbf{a}, \mathbf{b}$. We found that chloroquinones $\mathbf{1} \mathbf{a}, \mathbf{b}$ were only slightly soluble in $\mathrm{MeOH}$. In order to improve quinone solubility the mixture of methanol-acetone was used in subsequent experiments. The treatment of chloromethoxyquinone 1b with equimolar amount of thioglucose sodium salt 6 at r.t. for $1.5 \mathrm{~h}$ gave a mixture of methoxynaphthoquinone thioglucoside $4 \mathrm{~b}$ (56\% yield) and quinone tetracycle $\mathbf{5 a}$ (16\% yield) under $79 \%$ conversion of chloromethoxyquinone $\mathbf{1 b}$ (Scheme 2 ). Condensation of the dichloronaphthoquinone $1 \mathrm{a}(0.30 \mathrm{mM})$ with thioglucose sodium salt 6 (0.75 mM) proceeded easily within $30 \mathrm{~min}$, and led to dithioglucoside $4 \mathrm{a}$ in an excellent $93 \%$ yield.

The structures of the other starting quinones were chosen in such manner, that the heterocyclization of both diglycoside and related methoxymonoglucoside gave the same reaction product. The key dichloroquinones 7a,c were prepared by Friedel-Crafts condensation of dichloromaleic anhydride with suitable 1,4-hydroquinones ${ }^{15,16}$. The tetrachloroquinone $\mathbf{7 b}$ was obtained following literature procedure ${ }^{17}$. Methylation ${ }^{15}$ of quinones $7 \mathbf{a}, \mathbf{b}$ by $\mathrm{Mel} / \mathrm{Ag}_{2} \mathrm{O}$ in $\mathrm{CHCl}_{3}$ solution gave 5,8 -dimethoxychloroquinones $9 \mathbf{a}, \mathbf{b}$ in good $60-80 \%$ yield (Scheme 3). Partial substitution of one chlorine atom in 2,3-dichloroquinones 7a and 9a,b was achieved according to the literature ${ }^{15}$ by treatment with $\mathrm{AcONa} / \mathrm{MeOH}$ at reflux and led to methoxychloroquinones $\mathbf{8 a}, \mathbf{1 0 a , b}$ in good yields (75-85\%). Quinone 7c reacted with $\mathrm{AcONa} / \mathrm{MeOH}$ only by heating in an autoclave at $95{ }^{\circ} \mathrm{C}$ for $8 \mathrm{~h}$ and gave the desired product $8 \mathrm{~b}$ in a low $29 \%$ yield.

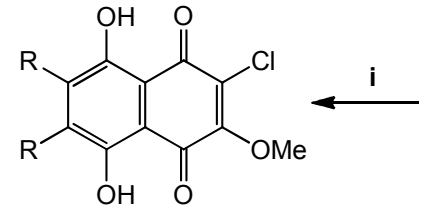

8a: $\mathrm{R}=\mathrm{H}$ 8b: $R=M e$<smiles>[R]c1c([R])c(O)c2c(c1O)C(=O)C(Cl)C(Cl)C2=O</smiles>

7a: $\mathrm{R}=\mathrm{H} \longrightarrow \mathrm{ii}$ 7b: $\mathrm{R}=\mathrm{Cl}$
7c: $\mathrm{R}=\mathrm{Me}$

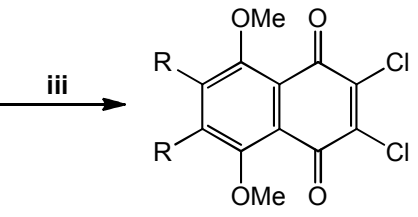

9a: $\mathrm{R}=\mathrm{H}$ 9b: $\mathrm{R}=\mathrm{Cl}$

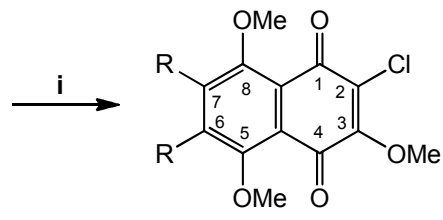

10a: $\mathrm{R}=\mathrm{H}$ 10b: $\mathrm{R}=\mathrm{Cl}$

Scheme 3. Reagents and conditions: (i) $\mathrm{AcONa} / \mathrm{MeOH}$, reflux; (ii) $\mathrm{HCl} / \mathrm{MnO}_{2}, \mathrm{AcOH}$, reflux; (iii) $\mathrm{Mel} / \mathrm{Ag}_{2} \mathrm{O}$, $\mathrm{CHCl}_{3}$, rt.

The condensation of dichloronaphthoquinones $9 \mathbf{a}, \mathbf{b}$, bearing chloro- and methoxysubstituents in quinoid core led to dithioglucosides $\mathbf{1 1} \mathbf{a}, \mathbf{b}$ with $89-94 \%$ yield. These dithioglucosides $\mathbf{1 1 a}, \mathbf{b}$ under treatment by $\mathrm{MeONa} / \mathrm{MeOH}$ were easily converted within 30-60 min in the tetracyclic conjugates $\mathbf{1 2 a , b}$ in good yields 
83-86\%. The substitution of chloromethoxyquinones 10a with equimolar ratio of thioglucose salt 6 proceeded in acetone/MeOH solution in $1 \mathrm{~h}$ and gave monoglucoside $14 \mathrm{a}$ in $80 \%$ yield. Under these conditions the trichloromethoxynaphthoquinone $\mathbf{1 0 b}$ led to monoglucoside $\mathbf{1 4 b}(\mathbf{7 4 \% )}$ and related tetracyclic conjugate $\mathbf{1 2 b}$ (7\%). Under MeONa/MeOH treatment, the monoglucosides $\mathbf{1 4 a , b}$ were readily converted to tetracycles $12 \mathbf{a}, \mathbf{b}$ in $75-81 \%$ yields. Tetracylic quinones $12 \mathrm{a}, \mathrm{b}$ were easily acetylated by $\mathrm{Ac}_{2} \mathrm{O} / \mathrm{Py}$ and gave acetyl derivatives $13 a, b$ in good yields $84-85 \%$.<smiles>[R]c1c([R])c2c(c(OC)c1OC)C(=O)C(Cl)=C(Cl)C2=O</smiles>

9a: $\mathrm{R}=\mathrm{H}$

9b: $\mathrm{R}=\mathrm{Cl}$<smiles>[R]c1c([R])c(OC)c2c(c1OC)C(=O)C(Cl)=C(OC)C2=O</smiles><smiles>[R]c1c([R])c(OC)c2c(c1OC)C(=O)C([Se])=C([SeH])C2=O</smiles>

11a: $\mathrm{R}=\mathrm{H}$ 11b: $\mathrm{R}=\mathrm{Cl}$

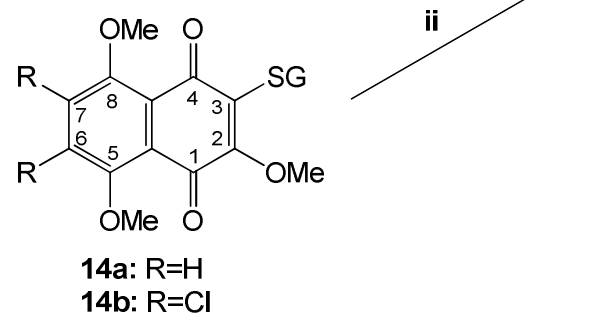

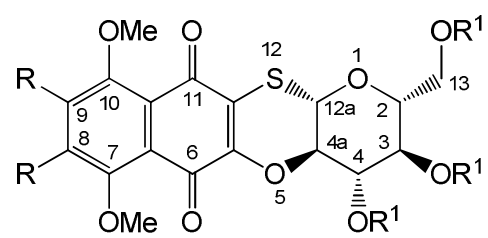

12a: $R=R^{1}=H$

12b: $\mathrm{R}=\mathrm{Cl}, \mathrm{R}^{1}=\mathrm{H} \quad \longrightarrow$ iii

13a: $R=H, R^{1}=A c$

13b: $\mathrm{R}=\mathrm{Cl}, \mathrm{R}^{1}=\mathrm{Ac}$

Scheme 4. Reagents and conditions: (i) acetone/MeOH, r.t.; (ii) $\mathrm{MeONa} / \mathrm{MeOH}$, r.t.; (iii) $\mathrm{Ac}_{2} \mathrm{O} / \mathrm{Py}$, rt.

The final part of the study was carried out on the 5,8-dihydroxy-1,4-naphthoquinone (naphthazarin) derivatives $\mathbf{7 a , b}$ and $\mathbf{8} \mathbf{a}, \mathbf{b}$. It is known that naphthazarin exists in various tautomeric forms that react with the formation of different reaction products ${ }^{15}$.

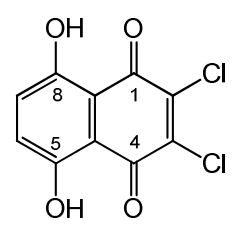

A<smiles>O=C1C=CC(=O)c2c(O)c(Cl)c(Cl)c(O)c21</smiles>

B

Scheme 5. Dichloronaphthazarin 7a tautomeric equilibrium.

To suppress the addition of thioglucose to the quinone ring of dichloronaphthazarin tautomer $B$ (Scheme 5), the dichloroquinone 7a $(0.50 \mathrm{mM})$ was condensed with reduced amount of thioglucose salt 6 $(0.91 \mathrm{mM})$ in acetone-methanol solution for $1 \mathrm{~h}$ and led to mixture of chloronaphthazarin thioglucoside 15a (21\%) and naphthazarin bisthioglucoside 15b (69 \%) (Scheme 6). Dichlorodimethylnaphthazarin $7 \mathrm{~b}$ (0.50 mM) reacted with an excess of thioglucose salt $6(1.261 \mathrm{mM})$ in only $18 \mathrm{~h}$ and gave the dithioglucoside dimethylnaphthazarin 15c in an excellent $95 \%$ yield. It can be assumed that the reduced reactivity of dichlorodimethylnaphthazarin $\mathbf{7 b}$ in nucleophilic substitution reaction is due to the electron donor effect of two methyl groups on tautomeric equilibrium of naphthazarin core. 

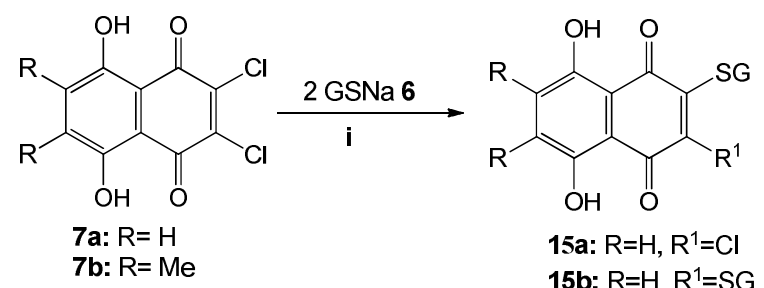

15a: $\mathrm{R}=\mathrm{H}, \mathrm{R}^{1}=\mathrm{Cl}$ 15b: $\mathrm{R}=\mathrm{H}, \mathrm{R}^{1}=\mathrm{SG}$ 15c: $R=M e, R^{1}=S G$

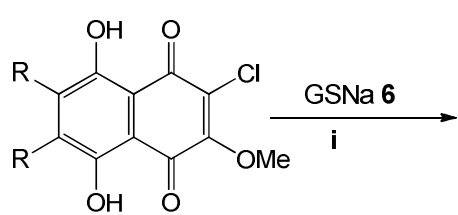

8a: $\mathrm{R}=\mathrm{H}$

8b: $R=M e$

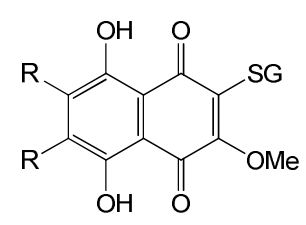

17a: $\mathrm{R}=\mathrm{H}$ 17b: $\mathrm{R}=\mathrm{Me}$

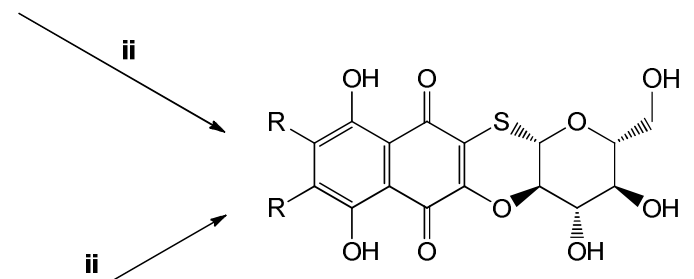

16a: $\mathrm{R}=\mathrm{H}$

16b: $R=M e$

Scheme 6. Reagents and conditions: (i) acetone/MeOH, r.t.; (ii) $\mathrm{MeONa/MeOH,} \mathrm{rt.}$

The substitution of chloromethoxynaphthoquinone 8 a $(0.40 \mathrm{mM})$ with thioglucose salt $6(0.50 \mathrm{mM})$ proceed in acetone/MeOH solution in $1.5 \mathrm{~h}$ until the disappearance of starting quinone $8 \mathrm{a}$ and formation of two polar products with $R_{f} 0.34$ and $R_{f} 0.10$. The first compound with $R_{f} 0.34$ was monoglucoside 17 a (80\%) and the polar product with $R_{f} 0.10$ was bisglucoside $15 \mathrm{c}(15 \%)$. The bisglucoside $15 \mathrm{c}$ was formed as a result of the replacement of the methoxy group in quinone $8 \mathbf{a}$. In these conditions the trichloromethoxynaphthoquinone $\mathbf{8 b}$ led to the formation of monoglucoside $\mathbf{1 7 b}(\mathbf{7 4 \% )}$ and related tetracycle conjugate $\mathbf{1 6 b}(7 \%)$. Under the base treatment by $\mathrm{MeONa} / \mathrm{MeOH}$ both monoglucosides $17 \mathbf{a}, \mathbf{b}$ were readily converted in tetracycles $\mathbf{1 6 a , b}$ with yields $75-81 \%$. It is evident, that tetracyclic quinone-glucoside conjugates of 12 a, $\mathbf{b}$ and $\mathbf{1 6 a , b}$ were formed from methoxymonoglucosides $14 \mathbf{a}, \mathbf{b}, \mathbf{1 7} \mathbf{a}, \mathbf{b}$ and bisglucosides 11a,b, 15b,c through intramolecular nucleophilic substitution of the methoxyl group or 1-thioglucose residue. This process proceeds with retention of the configuration of all asymmetric centers of the carbohydrate portion.

The structures of new compounds were proved by NMR, IR spectroscopy and HR mass spectrometry. Attachment of thioglucoside to naphthoquinone core in the new compounds was evidenced by appearance of the signals of thioglucose moiety together with the retention of other signals of aromatic protons, phenolic $\alpha$ hydroxyl groups of naphthazarin nucleus and the signals of methyl and methoxyl groups. The 1',2'-trans( $\beta$ )configuration of thioglucoside bond in naphthoquinone thioglucosides $\mathbf{4 a , b}, \mathbf{1 1} \mathbf{a}, \mathbf{b}, \mathbf{1 4 a , b}, \mathbf{1 5} \mathbf{a}, \mathbf{b}, \mathbf{c}$ and 17a,b was confirmed by the value of anomeric proton doublets $\left(J_{1^{\prime}, 2^{\prime}}=8.4-10.0 \mathrm{~Hz}\right)$ in the ${ }^{1} \mathrm{H} N M R$ spectra. Spectral data of new and known starting chloronaphthoquinones $\mathbf{7 a , b , c - 1 0 a , b}$ were in a good agreement with their proposed structures.

\section{Conclusions}

A novel and facile method for synthesis of base-sensitive di- and monothioglucosides of various 1,4naphthoquinones by the condensation of substituted chloronapthoquinones with the sodium salt of 1-thio- $\beta$ D-glucopyranose was developed. Both naphthoquinone di- and methoxymonothioglucosides under $\mathrm{MeONa} / \mathrm{MeOH}$ treatment were readily converted in linear tetracyclic quinone-carbohydrate conjugates in good yields. 
General. Melting points (uncorrected) were measured with a Boetius apparatus. IR spectra were recorded on Bruker Vector-22 FT-IR spectrophotometer. ${ }^{1} \mathrm{H}$ NMR spectra were recorded on a Bruker AVANCE-500 and Bruker AVANCE-700 at frequencies 500 and $700 \mathrm{MHz}$ for ${ }^{1} \mathrm{H}$ spectra and 125 and $176 \mathrm{MHz}$ for ${ }^{13} \mathrm{C}$ spectra, respectively. 2D NMR experiments $\left\{{ }^{1} \mathrm{H}-{ }^{1} \mathrm{H}\right\}$ COSY, $\left\{{ }^{1} \mathrm{H}-{ }^{13} \mathrm{C}\right\} \mathrm{HMBC}-\mathrm{qs}$, and $\left\{{ }^{1} \mathrm{H}^{-13} \mathrm{C}\right\} \mathrm{HSOC}$ were used where necessary in assigning NMR spectra. Spin-spin coupling constants $(J)$ were reported in hertz $(\mathrm{Hz})$. Chemical shifts were referenced to TMS $(\delta=0.00 \mathrm{ppm})$. El mass spectra and high resolution mass spectra were recorded on AMD-604S instrument at $70 \mathrm{eV}$. ESI mass spectra and ESI high resolution mass spectra were recorded on an Agilent 651 Q-TOF LC/MS instrument. 1-Thio- $\beta$-D-glucopyranose sodium salt (Chemapol) was used. Silufol UVVIS TLC plates (Chemapol) treated vapor of hydrochloric acid were used for analytical TLC. Preparative TLC was performed on silica gel 60 (Merck, 40-63 $\mu \mathrm{m}$ ). TLC was developed in system A: hexane-benzene-acetone, 2:1:1, system B: benzene-ethylacetate-methanol, 2:1:1 and system C: benzene-ethylacetate-methanol, 7:4:1.

Condensation of 2-chloro-3-methoxynaphthoquinone $1 \mathrm{~b}$ with thioglucose sodium salt 6. 2-Chloro-3methoxynaphthoquinone $1 \mathrm{~b} 111 \mathrm{mg}(0.50 \mathrm{mM})$ was dissolved in acetone $(10 \mathrm{~mL})$ and $\mathrm{MeOH}(10 \mathrm{~mL}), \beta-\mathrm{D}-$ thioglucopyranose sodium salt (6) $110 \mathrm{mg}(0.50 \mathrm{mM})$ was added. The resulting mixture was stirred during 1.5 $\mathrm{h}$ at room temperature until the consumption of thioglucose $\mathbf{6}$ and conversion of the starting quinone $1 \mathrm{~b}$ in the new yellow compound with $R_{f} 0.56$ (B). During the reaction, the formation of a brown precipitate was observed. The precipitate was filtered off, washed with water, acetone, dried in a vacuum and identified as $(2 R, 3 R, 4 S, 4 a R, 12 a S)$-2-Hydroxymethyl-3,4,-dihydroxy-3,4,4a,12a-tetrahydro-2H-naphtho[2,3-b]pyrano[2,3e][1,4]-oxathiine-6,11-dione (5a). Yield $28 \mathrm{mg}(16 \%)$, orange powder, mp 350-351 ${ }^{\circ} \mathrm{C}$ (Lit. data ${ }^{12}, \mathrm{mp} 350-351$ $\left.{ }^{\circ} \mathrm{C}\right)$. The filtrate was evaporated in vacuum and the residue was subjected to preparative TLC (system B) and yielded starting compound 2-chloro-3-methoxynaphthoquinone $\mathbf{1 b}(23 \mathrm{mg}, 21 \%)$ and 3-( $\mathbf{B}$-D-glucopyranosyl1-thio)-2-methoxynaphthalene-1,4-dione (4b). Yield $108 \mathrm{mg}$ (56\%), brown solid, mp 96-99 ${ }^{\circ} \mathrm{C} . R_{f} 0.56$ (B). ${ }^{1} \mathrm{H}$ NMR (700 MHz, DMSO- $\left.d_{6}\right): \delta 3.09\left(\mathrm{~m}, 3 \mathrm{H}, \mathrm{H}-2^{\prime}, 2 \mathrm{H}^{\prime} 4^{\prime}, \mathrm{H}-5^{\prime},\right)$ ), $3.22\left(\mathrm{~m}, 1 \mathrm{H}, \mathrm{H}-3^{\prime}\right), 3.34\left(\mathrm{~m}, 1 \mathrm{H}, \mathrm{H}-6^{\prime}\right), 3.53(\mathrm{~m}$, $\left.1 \mathrm{H}, \mathrm{H}^{-} 6^{\prime}\right), 4.11(\mathrm{~s}, 3 \mathrm{H}, \mathrm{ArOMe}), 4.33\left(\mathrm{t}, 1 \mathrm{H}, J 5.7 \mathrm{~Hz}, \mathrm{C}^{6^{\prime}} \mathrm{H}_{2} \mathrm{O} \underline{\mathrm{H}}\right), 4.93\left(\mathrm{~d}, 1 \mathrm{H}, J 4.4 \mathrm{~Hz}, \mathrm{C}^{4^{\prime}} \mathrm{HO} \underline{\mathrm{H}}\right), 5.10(\mathrm{~d}, 1 \mathrm{H}, J 4.0$ $\left.\mathrm{Hz}, \mathrm{C}^{3} \mathrm{HOH}\right), 5.29\left(\mathrm{~d}, 1 \mathrm{H}, J 9.7 \mathrm{~Hz}, \mathrm{H}-1^{\prime}\right), 5.46\left(\mathrm{~d}, 1 \mathrm{H}, J 6.2 \mathrm{~Hz}, \mathrm{C}^{2} \mathrm{HOH}\right), 7.82(\mathrm{~m}, 2 \mathrm{H}, \mathrm{ArH}), 7.95(\mathrm{~m}, 1 \mathrm{H}, \mathrm{ArH})$, $7.97(\mathrm{~m}, 1 \mathrm{H}, \mathrm{ArH}) .{ }^{13} \mathrm{C}$ NMR (DMSO- $\left.d_{6}, 176 \mathrm{MHz}\right): \delta 60.9$ (C-6'), $61.0(\mathrm{MeO}), 70.0\left(\mathrm{C}-4^{\prime}\right), 74.5\left(\mathrm{C}-2^{\prime}\right), 78.2\left(\mathrm{C}-3^{\prime}\right)$, 81.4 (C-5'), 82.6 (C-1'), 126.0, 130.9, 131.2, 132.0, 133.9, 157.8, 178.4, 182.5. IR (KBr): 3435 (OH), 2923, 1660 $(C=0), 1591,1555,1441,1385,1334,1254,1215,1142,1075,1046,1020,919 \mathrm{~cm}^{-1} . M S$ (ESI): $\mathrm{m} / \mathrm{z} 405$ [M+Na]. HRMS (ESI): calcd for $\mathrm{C}_{17} \mathrm{H}_{18} \mathrm{NaO}_{8} \mathrm{~S}$ 405.0615, found 405.0622 .

Condensation of 2,3-dichloronaphthoquinone (1a) with thioglucose sodium salt 6. 2,3Dichloronaphthoquinone (1a) $68 \mathrm{mg}(0.35 \mathrm{mM})$ was dissolved in acetone $(6 \mathrm{~mL})$ and $\mathrm{MeOH}(6 \mathrm{~mL})$ and glucose sodium salt $6163 \mathrm{mg}(0.75 \mathrm{mM})$ was added. The resulting mixture was stirred during $1.5 \mathrm{~h}$ at room temperature until the conversion of starting quinone $1 \mathrm{a}$ with $R_{f} 0.90$ (B) into a new yellow compound with $R_{f}$ 0.30 (B). Inorganic salts were filtered off, and the precipitate was washed with acetone. The combined filtrate was evaporated in vacuum, and the residue was subjected to preparative TLC, yielded 2,3-bisglucoside 2,3di(B-D-glucopyranosyl-1-thio)naphthalene-1,4-dione (4a). Yield $152 \mathrm{mg}(93 \%)$, yellow powder, $R_{f} 0.30(\mathrm{~B}), \mathrm{mp}$ $>360^{\circ} \mathrm{C} .{ }^{1} \mathrm{H}$ NMR $\left(700 \mathrm{MHz}\right.$, DMSO- $\left.d_{6}\right): \delta 3.04\left(\mathrm{~m}, 2 \mathrm{H}, 2 \times \mathrm{H}^{-} 5^{\prime}\right), 3.10\left(\mathrm{~m}, 2 \mathrm{H}, 2 \times \mathrm{H}-4^{\prime}\right), 3.14\left(\mathrm{~m}, 2 \mathrm{H}, 2 \times \mathrm{H}-2^{\prime}\right)$, $3.33\left(\mathrm{~m}, 2 \mathrm{H}, 2 \times \mathrm{H}^{-} 6^{\prime}\right), 3.46\left(\mathrm{~m}, 2 \mathrm{H}, 2 \times \mathrm{H}^{-} 6^{\prime}\right), 4.26\left(\mathrm{~m}, 2 \mathrm{H}, 2 \times \mathrm{C}^{6^{\prime}} \mathrm{H}_{2} \mathrm{OH}\right), 4.93\left(\mathrm{~m}, 2 \mathrm{H}, 2 \times \mathrm{C}^{4} \mathrm{HOH}\right), 5.10(\mathrm{~m}, 2 \mathrm{H}, 2$ $\left.\times \mathrm{C}^{3^{\prime}} \mathrm{HOH}\right), 5.37\left(\mathrm{~d}, 2 \mathrm{H}, J 9.4 \mathrm{~Hz}, 2 \times \mathrm{H}-1^{\prime}\right), 5.49\left(\mathrm{~m}, 2 \mathrm{H}, 2 \times \mathrm{C}^{2} \mathrm{HOH}\right), 7.79(\mathrm{~m}, 2 \mathrm{H}, \mathrm{H}-6, \mathrm{H}-7), 7.94(\mathrm{~m}, 2 \mathrm{H}, \mathrm{H}-5, \mathrm{H}-$ 8). ${ }^{13} \mathrm{C}$ NMR (DMSO-d $6,176 \mathrm{MHz}$ ): $\delta 60.7$ (C-6'), 69.9 (C-4'), 74.9 (C-2'), 78.2 (C-3'), 81.6 (C-5'), 83.9 (C-1'), 
126.5, 132.6, 133.7, 146.5, 178.7 (C=O). IR (KBr): 3425 (OH), 2926, 1658 (C=O), 1617, 1414, 1275, 1181, 1141, 1075, $1049 \mathrm{~cm}^{-1}$. MS (ESI): $\mathrm{m} / z 569$ [M+Na]. HRMS (ESI): calcd for $\mathrm{C}_{22} \mathrm{H}_{26} \mathrm{NaO}_{12} \mathrm{~S}_{2}$ 569.0758, found 569.0752 .

2-Chloro-5,8-dihydroxy-3-methoxynaphtalene-1,4-dione (8a). 2,3-Dichloroquinone 7a $259 \mathrm{mg}$ (1.00 mM), dry AcONa $790 \mathrm{mg}(9.61 \mathrm{mM})$ and dry methanol $(60 \mathrm{~mL})$ was stirred at reflux $0.5 \mathrm{~h}$. Reaction mixture was cooled, was acidified in drops with conc. $\mathrm{HCl}$, inorganic salts were filtrated off, the precipitate was washed with acetone and filtrate was evaporated in vacuum. The crystallization of residue from MeOH gave $185 \mathrm{mg}$ (72\%) of 2-chloro-5,8-dihydroxy-3-methoxynaphtalene-1,4-dione (8a); red solid, mp $160-162^{\circ} \mathrm{C}$, (lit. data ${ }^{17} 161-$ $\left.162^{\circ} \mathrm{C}\right)$.

2-Chloro-5,8-dihydroxy-3-methoxy-6,7-dimethylnaphtalene-1,4-dione (8b). 2,3-Dichloroquinone $7 \mathrm{~b} 287 \mathrm{mg}$ $(1.00 \mathrm{mM})$, dry AcONa $790 \mathrm{mg}(9.61 \mathrm{mM})$ and dry methanol $(100 \mathrm{~mL})$ was stirred in stainless autoclave at $95^{\circ} \mathrm{C}$ within $5 \mathrm{~h}$. The reaction mixture was cooled, acidified in drops with conc. $\mathrm{HCl}$, inorganic salts were filtrated off, and the filtrate was evaporated in vacuum. The residue was subjected to preparative TLC on silica gel, eluting with system $\mathrm{A}$, to give polar red fraction with $R_{f} 0.36(\mathrm{~A})$. The crystallization of the fraction from $\mathrm{MeOH}$ yielded $82 \mathrm{mg}$ (29\%) of 2-chloro-5,8-dihydroxy-3-methoxy-6,7-dimethylnaphtalene-1,4-dione (8b); red solid, $\mathrm{mp}$ 188$190{ }^{\circ} \mathrm{C} .{ }^{1} \mathrm{H}$ NMR $\left(500 \mathrm{MHz}, \mathrm{CDCl}_{3}\right): \delta 2.26$ (s, 3H, ArMe), 2.27 (s, 3H, ArMe), 4.27 (s, 3H, OMe), 13.06 (s, $1 \mathrm{H}, \alpha-$ $\mathrm{OH}), 13.25$ (s, 1H, $\alpha-\mathrm{OH}) .{ }^{13} \mathrm{C} \mathrm{NMR}\left(\mathrm{CDCl}_{3}, 125 \mathrm{MHz}\right): \delta 12.4$ (Me), 12.6 (Me), 61.9 (OMe), 107.5, 109.0, 127.3, 140.0, 141.1, 156.2, 165.9, 166.9, 173.3 (C=O), 174.0 (C=O). IR ( $\left.\mathrm{CHCl}_{3}\right): 2951,1606$ (C=0), 1577, 1448, 1408, $1393,1285,1265,1200,1182,1155,1043 \mathrm{~cm}^{-1}$. HRMS (EI): calcd for $\mathrm{C}_{13} \mathrm{H}_{11} \mathrm{O}_{5} \mathrm{Cl} 282.0295$ found 282.0308.

2,3-Dichloro-5,8-dimethoxynaphthalene-1,4-dione (9a). Dichloronaphthazarine 7a was methylated by $\mathrm{CH}_{3} \mathrm{l} / \mathrm{Ag}_{2} \mathrm{O}$ according Brassard procedure ${ }^{15}$ and yielded 2,3-dichloro-5,8-dimethoxynaphthalene-1,4-dione (9a); red solid, mp: $236-237^{\circ} \mathrm{C}$, (lit. data ${ }^{15}$, mp: $237-238^{\circ} \mathrm{C}$ ).

2,3,6,7-Tetrachloro-5,8-dimethoxynaphthalene-1,4-dione (9b). ${ }^{18} \mathrm{~A}$ mixture of tetrachloronaphthazarine $\mathbf{7 b}$ (6.72 g, $0.02 \mathrm{M}), \mathrm{CH}_{3} \mathrm{l}(13 \mathrm{~mL})$, and $\mathrm{Ag}_{2} \mathrm{O}(10.0 \mathrm{~g})$, was stirred at room temperature. The same amounts of $\mathrm{CH}_{3} \mathrm{l}$ and $\mathrm{Ag}_{2} \mathrm{O}$ were added after 6 and $16 \mathrm{~h}$ of the reaction, and the reaction mixture was stirred $29 \mathrm{~h}$ until complete conversion of red quinone $\mathbf{7 b}, R_{f} 0.80(\mathrm{~A})$, into yellow dimethoxyquinone $9 \mathrm{~b}, R_{f} 0.75$ (A). Inorganic salts were filtered off, the residue was washed with $\mathrm{CHCl}_{3}$, combined filtrate was evaporated, and the residue was crystallized from $\mathrm{CHCl}_{3}$ to give 2,3,6,7-tetrachloro-5,8-dimethoxynaphthalene-1,4-dione (9b). Yield 5.60 g (80\%), beige powder, mp: $208-210{ }^{\circ} \mathrm{C} .{ }^{1} \mathrm{H} \mathrm{NMR}\left(500 \mathrm{MHz}, \mathrm{CDCl}_{3}\right): 4.00(6 \mathrm{H}, \mathrm{s}, 2 \times \mathrm{OMe}) .{ }^{13} \mathrm{C} \mathrm{NMR}(125 \mathrm{MHz}$, $\mathrm{CDCl}_{3}$ ): 62.1 (2), 123.3 (2), 138.1 (2), 143.0 (2), 154.2 (2), 173.7 (2). IR ( $\left.\mathrm{CHCl}_{3}\right): 3014,2981,2856,1687$ (C=O), 1586, 1524, 1379, 1321, 1201, 1163, 1054, $1029 \mathrm{~cm}^{-1} . \mathrm{MS}(\mathrm{El}, 70 \mathrm{eV}), \mathrm{m} / \mathrm{z}(\%): 356\left(\mathrm{M}^{+}, 100\right), 339(42), 291$ (42), 255 (36), 233 (59), 205 (27), 155 (21), 123 (21), 87 (87), 32 (83). HRMS (El): calcd 353.9020 for $\mathrm{C}_{12} \mathrm{H}_{6} \mathrm{Cl}_{4} \mathrm{O}_{4}$, found 353.9034 .

3-Chloro-2,5,8-trimethoxyaphthalene-1,4-dione (10a) and 2,6,7-trichloro-3,5,8-trimethoxynaphtalene-1,4dione (10b). 2,3-Dichloroquinone 9a,b (1.00 mM), dry AcONa $400 \mathrm{mg}(2.40 \mathrm{mM})$ and dry methanol (30 mL) were stirred at reflux within $15 \mathrm{~h}$ for $(9 \mathrm{a})$ and $5 \mathrm{~h}$ for $(9 \mathrm{~b})$. The hot reaction mixture was filtrated off from inorganic salts and then was cooled at $+5{ }^{\circ} \mathrm{C}$. Upon filtration methoxychloroquinones 10a,b were obtained: 3chloro-2,5,8-trimethoxyaphthalene-1,4-dione (10a), yield $238 \mathrm{mg}(85 \%)$, yellow needles, $R_{f} 0.39(\mathrm{~A}), \mathrm{mp} 144-$ $146{ }^{\circ} \mathrm{C}$. (lit. data ${ }^{19}, \mathrm{mp} 146-148{ }^{\circ} \mathrm{C}$ ); 2,6,7-trichloro-3,5,8-trimethoxynaphtalene-1,4-dione (10b), yield $263 \mathrm{mg}$ (75\%), yellow needles, $R_{f} 0.41(\mathrm{~A}), \mathrm{mp} 156-158{ }^{\circ} \mathrm{C} .{ }^{1} \mathrm{H} \mathrm{NMR}\left(500 \mathrm{MHz}, \mathrm{CDCl}_{3}\right)$ : $\delta 3.97$ (s, 3H, OMe), 3.99 (s, 3H, OMe), 4.25 (s, 3H, OMe). $\left.{ }^{13} \mathrm{C} \mathrm{NMR} \mathrm{(CDCl}, 125 \mathrm{MHz}\right): \delta 61.5$ (OMe), 62.0 (OMe), 62.2 (OMe), 123.4, 123.7, 127.2, 136.9, 137.6, 153.4, 153.5, 156.7, 176.2 (C=O), 177.4 (C=O). IR ( $\left.\mathrm{CHCl}_{3}\right): 2944,1676$ (C=O), 1603, 1525, $1459,1380,1331,1307,1117,1029 \mathrm{~cm}^{-1}$. HRMS (EI): calcd for $\mathrm{C}_{13} \mathrm{H}_{9} \mathrm{O}_{5} \mathrm{Cl}_{3} 349.9515$ found 349.9532.

2,3-Diglucosides 11a,b. 2,3-Dichloronaphthoquinone 9a,b $(0.50 \mathrm{mM})$ was dissolved in acetone $(20 \mathrm{~mL})$ and $\mathrm{MeOH}(20 \mathrm{~mL})$ and glucose sodium salt $6275 \mathrm{mg}(1.26 \mathrm{mM})$ was added. The resulting mixture was stirred 
during $1.5 \mathrm{~h}$ at room temperature until the conversion of starting quinone $9 \mathrm{a}, \mathrm{b}$ with $R_{f} 0.90-0.95$ (B) into a new yellow compound with $R_{f} 0.27-0.28(\mathrm{~B})$. Inorganic salts were filtered off, and the precipitate was washed with acetone. The combined filtrate was evaporated in vacuum, and the residue was subjected to preparative TLC and led to 2,3-bisglucosides 11a,b.

2,3-Di( $\beta$-D-glucopyranosyl-1-thio)-5,8-dimethoxynaphthalene-1,4-dione (11a). Yield $287 \mathrm{mg}$ (94\%), amorphous brown powder, $R_{f} 0.27$ (B), $\mathrm{mp}>360{ }^{\circ} \mathrm{C} .{ }^{1} \mathrm{H} \mathrm{NMR}\left(500 \mathrm{MHz}, \mathrm{DMSO}-d_{6}\right): \delta 3.00\left(\mathrm{~m}, 2 \mathrm{H}, 2 \times \mathrm{H}-5^{\prime}\right)$, $3.18\left(\mathrm{~m}, 6 \mathrm{H}, 2 \times \mathrm{H}^{-2} \mathrm{C}^{\prime}, 2 \times \mathrm{H}-3^{\prime}, 2 \times \mathrm{H}^{\prime} \mathbf{4}^{\prime}\right), 3.42\left(\mathrm{~m}, 4 \mathrm{H}, 4 \times \mathrm{H}-6^{\prime}\right), 3.80(\mathrm{~s}, 6 \mathrm{H}, 2 \times \mathrm{OMe}), 4.24(\mathrm{br} . \mathrm{s}, 2 \mathrm{H}, 2 \times$ $\mathrm{C}^{6} \mathrm{H}_{2} \mathrm{O} \underline{\mathrm{H}}$ ), 4.93 (br.s, $\left.2 \mathrm{H}, 2 \times \mathrm{C}^{4} \mathrm{HO} \underline{\mathrm{H}}\right), 5.10$ (br.s, $2 \mathrm{H}, 2 \times \mathrm{C}^{3^{\prime}} \mathrm{HO} \underline{\mathrm{H}}$ ), 5.12 (d, 2H, J $\left.9.2 \mathrm{~Hz}, 2 \times \mathrm{H}-1^{\prime}\right), 5.44$ (br.s, $2 \mathrm{H}, 2$ $\left.\times \mathrm{C}^{2^{\prime}} \mathrm{HO} \underline{\mathrm{H}}\right), 7.41(\mathrm{~s}, 2 \mathrm{H}, \mathrm{H}-6, \mathrm{H}-7) .{ }^{13} \mathrm{C}$ NMR (DMSO-d, $\left.125 \mathrm{MHz}\right): \delta 56.62(\mathrm{MeO}), 60.25\left(\mathrm{C}-6{ }^{\prime}\right), 69.30$ (C-4'), 74.48 (C-2'), 78.16 (C-3'), 81.31 (C-5'), 83.59 (C-1'), 119.64, 122.16, 144.68, 151.93, $178.04(\mathrm{C}=0) . \mathrm{IR}(\mathrm{KBr})$ : $3420(\mathrm{OH}), 2922,1667$ (C=O), 1592, 1568, 1525, 1481, 1435, 1414, 1278, 1257, 1211, 1181, 1050, $933 \mathrm{~cm}^{-1} . \mathrm{MS}$ (ESI): $m / z 629$ [M+Na]. HRMS (ESI): calcd for $\mathrm{C}_{24} \mathrm{H}_{30} \mathrm{NaO}_{14} \mathrm{~S}_{2} 629.0969$, found 629.0971 .

6,7-Dichloro-2,3-di( $\beta$-D-glucopyranosyl-1-thio)-5,8-dimethoxynaphthalene-1,4-dione (11b). Yield $301 \mathrm{mg}$ (89\%), brown solid, $R_{f} 0.28(\mathrm{~B}), \mathrm{mp} 151-152^{\circ} \mathrm{C} .{ }^{1} \mathrm{H}$ NMR $\left(700 \mathrm{MHz}, \mathrm{DMSO}-d_{6}\right): \delta 3.03\left(\mathrm{~m}, 2 \mathrm{H}, 2 \times \mathrm{H}-5^{\prime}\right), 3.15(\mathrm{~m}$, $\left.6 \mathrm{H}, 2 \times \mathrm{H}^{-2} \mathrm{C}^{\prime}, 2 \times \mathrm{H}^{-3} \mathrm{3}^{\prime}, 2 \times \mathrm{H}-4^{\prime}\right), 3.39\left(\mathrm{~m}, 2 \mathrm{H}, 2 \times \mathrm{H}-6^{\prime}\right), 3.48\left(\mathrm{~m}, 2 \mathrm{H}, 2 \times \mathrm{H}-6^{\prime}\right), 3.87(\mathrm{~s}, 6 \mathrm{H}, 2 \times \mathrm{OMe}), 4.30$ (br.s, $2 \mathrm{H}, 2 \times \mathrm{C}^{6} \mathrm{H}_{2} \mathrm{OH}$ ), 4.94 (d, $\left.2 \mathrm{H}, J 5.2 \mathrm{~Hz}, 2 \times \mathrm{C}^{4} \mathrm{HO} \underline{\mathrm{H}}\right), 5.11$ (br.s, $\left.2 \mathrm{H}, 2 \times \mathrm{C}^{3} \mathrm{HO} \underline{\mathrm{H}}\right), 5.15$ (d, $\left.2 \mathrm{H}, J 9.1 \mathrm{~Hz}, 2 \times \mathrm{H}-1^{\prime}\right)$, 5.51 (br.s, $\left.2 \mathrm{H}, J 5.5 \mathrm{~Hz}, 2 \times \mathrm{C}^{2} \mathrm{HOH}\right) .{ }^{13} \mathrm{C} \mathrm{NMR}$ (DMSO-d, $175 \mathrm{MHz}$ ): $\delta 60.5$ (C-6'), 62.3 (MeO), 69.5 (C-4'), 74.8 (C-2'), 78.1 (C-3'), 81.6 (C-5'), 83.6 (C-1'), 126.3, 133.8, 145.4, 151.7, 176.5. IR (KBr): 3403 (OH), 2936,1672 (C=O), 1521, 1459, 1382, 1317, 1195, 1166, 1046, 1023, 877, 808, $\mathrm{cm}^{-1}$. MS (ESI): $\mathrm{m} / \mathrm{z} 697$ [M+Na]. HRMS (ESI): calcd for $\mathrm{C}_{24} \mathrm{H}_{28} \mathrm{Cl}_{2} \mathrm{NaO}_{14} \mathrm{~S}_{2}$ 697.0190, found 697.0181.

Tetracyclic quinones $12 \mathbf{a}, \mathbf{b}$. 2,3-Dichloronaphthoquinone $\mathbf{9 a , b}(0.50 \mathrm{mM})$ was dissolved in mixture of acetone $(20 \mathrm{~mL})$ and $\mathrm{MeOH}(20 \mathrm{~mL})$ and thioglucose sodium salt $6275 \mathrm{mg}(1.26 \mathrm{mM})$ was added and stirred 15-20 min until complete conversion quinone $\mathbf{9 a , b}$ in dithioglucoside $\mathbf{1 1} \mathbf{a}, \mathbf{b}$. The reaction mixture was evaporated under reduced pressure to remove acetone. The residue was dissolved in $\mathrm{MeOH}(15 \mathrm{~mL})$ and $0.5 \mathrm{~N} \mathrm{MeONa/MeOH}$ $(0.6 \mathrm{~mL}, 0.3 \mathrm{mM})$ was added. The mixture was kept at room temperature until TLC analysis indicated complete consumption of $\mathbf{1 1 a , b}$ (after $1 \mathrm{~h}$ ) and formation new compound 12a,b. During the reaction of $\mathbf{9 a}$, the formation of a brown precipitate 12a was observed. The precipitate was filtered of, washed with water, dry $\mathrm{MeOH}$ and gave high purity quinone $\mathbf{1 2 a}$. The reaction mixture with quinone $\mathbf{1 2 b}$ was concentrated further and subjected preparative TLC; double development with system B gave orange band of quinone 12b. The quinone $12 b$ was eluted from $\mathrm{SiO}_{2}$ with acetone and equal volume of $\mathrm{MeOH}$ was added to acetone eluate. Gently evaporation of this solution on reduced pressure led to formation pure orange solid of tetracyclic quinone $12 b$.

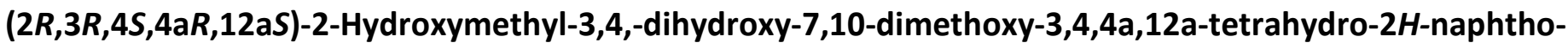
[2,3-b]pyrano[2,3-e][1,4]-oxathiine-6,11-dione (12a). Yield $176 \mathrm{mg}(86 \%)$, red solid, $R_{f} 0.50$ (C), mp 332-335 ${ }^{\circ} \mathrm{C} .{ }^{1} \mathrm{H}$ NMR $\left(700 \mathrm{MHz}, \mathrm{DMSO}-d_{6}\right): \delta 3.29(\mathrm{~m}, 1 \mathrm{H}, \mathrm{H}-3), 3.48(\mathrm{~m}, 3 \mathrm{H}, \mathrm{H}-2, \mathrm{H}-4 \mathrm{a}, \mathrm{H}-13) 3.57(\mathrm{~m}, 1 \mathrm{H}, \mathrm{H}-4), 3.74(\mathrm{~m}$, $1 \mathrm{H}, \mathrm{H}-13$ ), 3.85 (s, 3H, ArOMe), 3.86 (s, 3H, ArOMe), 4.72 (br.s, 1H, $\mathrm{C}^{13} \mathrm{H}_{2} \mathrm{O} \underline{\mathrm{H}}$ ), 4.92 (d, $1 \mathrm{H}$, J 8.5 Hz, H-12a), 5.37 (br.s, $1 \mathrm{H}, \mathrm{C}^{3} \mathrm{HO} \underline{\mathrm{H}}$ ), 5.58 (br.s, $\left.1 \mathrm{H}, \mathrm{C}^{4} \mathrm{HO} \underline{\mathrm{H}}\right), 7.52$ (d, $\left.1 \mathrm{H}, J 9.7 \mathrm{~Hz}, \mathrm{ArH}\right), 7.54$ (d, $\left.1 \mathrm{H}, J 9.7 \mathrm{~Hz}, \mathrm{ArH}\right) .{ }^{13} \mathrm{C} \mathrm{NMR}$ (DMSO-d $d_{6}, 176 \mathrm{MHz}$ ): $\delta 56.7$ (OMe), 56.8 (OMe), 60.8 (C-13), 70.5 (C-3), 73.7 (C-12a), 73.9 (C-4), 79.2 (C-4a), 82.2 (C-2), 118.6, 118.9, 121.7, 122.0, 122.4, 149.7, 153.3, 153.8, 174.7 (C=0), $179.9(\mathrm{C}=\mathrm{O}) . \mathrm{IR}(\mathrm{KBr}) 3444(\mathrm{OH})$, 1638 (C=O), 1614, 1561, 1476, 1405, 1266, 1181, 1075, $935 \mathrm{~cm}^{-1}$. MS (ESI): $\mathrm{m} / z \quad 433$ [M+Na]. HRMS (ESI): calcd for $\mathrm{C}_{18} \mathrm{H}_{18} \mathrm{NaO}_{9} \mathrm{~S} 433.0564$, found 433.0562 .

(2R,3R,4S,4aR,12aS)-8,9-Dichloro-2-hydroxymethyl-3,4-dihydroxy-7,10-dimethoxy-3,4,4a,12a-tetrahydro2H-naphtho[2,3-b]pyrano[2,3-e][1,4]-oxathiine-6,11-dione (12b). Yield $198 \mathrm{mg}(83 \%)$, orange solid, $R_{f} 0.28$ (C), mp 222-224 ${ }^{\circ} \mathrm{C} .{ }^{1} \mathrm{H}$ NMR (500 MHz, DMSO- $\left.d_{6}\right): \delta 3.32(\mathrm{~m}, 1 \mathrm{H}, \mathrm{H}-3), 3.50(\mathrm{~m}, 2 \mathrm{H}, \mathrm{H}-2, \mathrm{H}-13) ,3.54(\mathrm{~m}, 1 \mathrm{H}, \mathrm{H}-$ 
4a), $3.60(\mathrm{~m}, 1 \mathrm{H}, \mathrm{H}-4), 3.75(\mathrm{~m}, 1 \mathrm{H}, \mathrm{H}-13), 3.82$ (s, 3H, ArOMe), 3.83 (s, 3H, ArOMe), $4.74\left(\mathrm{~m}, 1 \mathrm{H}, \mathrm{C}^{13} \mathrm{H}_{2} \mathrm{O} \underline{\mathrm{H}}\right)$, $4.96(\mathrm{~d}, 1 \mathrm{H}, J 8.0 \mathrm{~Hz}, \mathrm{H}-12 \mathrm{a}), 5.40$ (d, $\left.1 \mathrm{H}, J 5.9 \mathrm{~Hz}, \mathrm{C}^{3} \mathrm{HO} \underline{\mathrm{H}}\right), 5.67$ (d, $\left.1 \mathrm{H}, J 5.9 \mathrm{~Hz}, \mathrm{C}^{4} \mathrm{HO} \underline{\mathrm{H}}\right) .{ }^{13} \mathrm{C}$ NMR (DMSO- $d_{6}$, $125 \mathrm{MHz}$ ): $\delta 60.7$ (C-13), 61.5 (2OMe), 70.4 (C-3), 73.6 (C-12a), 73.9 (C-4), 79.3 (C-4a), 82.2 (C-2), 123.1, 123.4, 123.9, 135.1, 135.3, 149.9, 152.3, 152.9, 173.4 (C=0), 178.9 (C=O). IR (KBr): 3432, 2941, 1653, 1603, 1625, 1458, 1381, 1333, 1275, 1209, 1131, 1025, $951 \mathrm{~cm}^{-1}$. MS (ESI): $\mathrm{m} / z 500$ [M+Na]. HRMS (ESI): calcd for $\mathrm{C}_{18} \mathrm{H}_{16} \mathrm{Cl}_{2} \mathrm{NaO}_{9} \mathrm{~S} 500.9784$, found 500.9784 .

Acetylation of tetracycles $\mathbf{1 2 a}, \mathbf{b}$ by $\mathrm{Ac}_{2} \mathrm{O} / \mathrm{Py}$. Quinone $\mathbf{1 2 a}, \mathbf{b}(0.20 \mathrm{mM})$ was dissolved in $\mathrm{Py}(3.0 \mathrm{~mL}), \mathrm{Ac}_{2} \mathrm{O}(1.5$ $\mathrm{mL}$ ) was added and stand overnight at room temperature. The reaction mixture was poured into ice, the precipitate was triturated with ice before crystals formation. The crystals were filtered off, washed with diluted $\mathrm{HCl}$, water, dried, recrystallized from $\mathrm{MeOH}$-benzene to give:

(2R,3R,4S,4aR,12aS)-2-Acetoxymethyl-3,4,-diacetoxy-7,10-dimethoxy-3,4,4a,12a-tetrahydro-2H-naphtho[2,3-b]pyrano[2,3-e][1,4]-oxathiine-6,11-dione (13a). Yield $91 \mathrm{mg}(85 \%)$, orange solid, mp $235-237{ }^{\circ} \mathrm{C} .{ }^{1} \mathrm{H}$ NMR (700 MHz, DMSO- $d_{6}$ ): $\delta 2.02$ (s, 3H, AcO), 2.04 (s, 3H, AcO), 2.08 (s, 3H, AcO), 3.85 (s, 3H, ArOMe), 3.86 (s, 3H, ArOMe), 3.99 (dd, 1H, J 8.4, $9.6 \mathrm{~Hz}, \mathrm{H}-4 \mathrm{a}), 4.10$ (dd, 1H, J 2.2, $12.6 \mathrm{~Hz}, \mathrm{H}-13$ ), 4.18 (dd, 1H, J 5.5, $12.6 \mathrm{~Hz}$, $\mathrm{H}-13$ ), 4.23 (ddd, 1H, J 2.2, 5.5, $9.6 \mathrm{~Hz}, \mathrm{H}-2$ ), 5.07 (dd, $1 \mathrm{H}, J 9.6 \mathrm{~Hz}, \mathrm{H}-3$ ), 5.28 (d, $1 \mathrm{H}, J 8.4 \mathrm{~Hz}, \mathrm{H}-12 \mathrm{a}$ ), 5.53 (dd, $1 \mathrm{H}, J 9.6 \mathrm{~Hz}, \mathrm{H}-4), 7.54(\mathrm{~d}, 1 \mathrm{H}, J 9.5 \mathrm{~Hz}, \mathrm{ArH}), 7.56$ (d, 1H, J $9.5 \mathrm{~Hz}, \mathrm{ArH}) .{ }^{13} \mathrm{C} N M R\left(\mathrm{DMSO}-d_{6}, 176 \mathrm{MHz}\right): \delta 20.4$ $\left(\underline{\mathrm{CH}}_{3} \mathrm{CO}\right), 20.5\left(\underline{\mathrm{C}}_{3} \mathrm{CO}\right), 20.6\left(\underline{\mathrm{CH}}_{3} \mathrm{CO}\right), 56.67$ (OMe), 56.70 (OMe), 61.8 (C-13), 68.3 (C-3), 71.7 (C-4), 73.2 (C12a), 75.5 (C-5), 75.7 (C-2), 118.3, 118.6, 121.8, 122.0, 122.5, 148.6, 153.4, 153.9, 169.3 ( $\left.\mathrm{CH}_{3} \mathrm{C} \mathrm{O}\right), 169.4$ $\left(\mathrm{CH}_{3} \underline{\mathrm{CO}}\right), 170.1$ ( $\left.\mathrm{CH}_{3} \underline{\mathrm{CO}}\right), 174.1$ ( $\left.\mathrm{C}=0\right), 179.6$ (C=O). IR $\left(\mathrm{CHCl}_{3}\right):$ 2941, $1753\left(\mathrm{CH}_{3} \mathrm{COOR}\right), 1664(\mathrm{C}=0), 1651,1613$, 1567, 1478, 1463, 1435, 1408, 1370, 1341, 1267, 1098, 1065, $1031 \mathrm{~cm}^{-1}$. HRMS (ESI, m/z): [M-H] calcd 535.0916 for $\mathrm{C}_{24} \mathrm{H}_{24} \mathrm{O}_{12} \mathrm{~S}$ found 535.0908 , and

(2R,3R,4S,4aR,12aS)-2-Acetoxymethyl-3,4,-diacetoxy-8,9-dichloro-7,10-dimethoxy-3,4,4a,12a-tetrahydro2H-naphtho[2,3-b]pyrano[2,3-e][1,4]-oxathiine-6,11-dione (13b). Yield $102 \mathrm{mg}$ (84\%), orange solid, mp 159$162{ }^{\circ} \mathrm{C} .{ }^{1} \mathrm{H}$ NMR (700 MHz, DMSO-d 6 ): $\delta 2.07$ (s, 3H, AcO), 2.11 (s, 3H, AcO), 2.16 (s, 3H, AcO), 3.88 (dd, 1H, J 8.4, 9.7 Hz, H-4a), 3.93 (ddd, $1 \mathrm{H}, J$ 2.2, 4.9, $9.5 \mathrm{~Hz}, \mathrm{H}-2$ ), 3.94 (s, 6H, ArOMe), 4.20 (dd, 1H, J 2.2, 12.6 Hz, H-13), 4.30 (dd, 1H, J 4.9, $12.6 \mathrm{~Hz}, \mathrm{H}-13), 4.84$ (d, 1H, J $8.4 \mathrm{~Hz}, \mathrm{H}-12 \mathrm{a}), 5.22$ (dd, 1H, J 9.7, $9.8 \mathrm{~Hz}, \mathrm{H}-3$ ), 5.45 (dd, $1 \mathrm{H}, J$ 9.5, 9.7 Hz, H-4). ${ }^{13} \mathrm{C}$ NMR (DMSO-d, $\left.176 \mathrm{MHz}\right): \delta 20.6\left(\underline{\mathrm{C}}_{3} \mathrm{CO}\right), 20.7\left(2 \times \underline{\mathrm{CH}}_{3} \mathrm{CO}\right), 61.7$ (C-13), 61.9 (OMe), 62.0 (OMe), 68.3 (C-3), 72.2 (C-4), 74.6 (C-12a), 76.5 (C4a), 77.2 (C-2), 122.8, 123.4, 124.6, 137.3, 137.5, 149.0, 153.5, 154.0, 169.4 ( $\left.\mathrm{CH}_{3} \underline{\mathrm{CO}}\right), 170.0$ ( $\left.\mathrm{CH}_{3} \underline{\mathrm{CO}}\right), 170.5\left(\mathrm{CH}_{3} \underline{\mathrm{CO}}\right), 173.0$ (C=0),178.8 (C=O). IR ( $\left.\mathrm{CHCl}_{3}\right): 2943,1754$ $\left(\mathrm{CH}_{3} \mathrm{CO}_{2} \mathrm{R}\right), 1672(\mathrm{C}=\mathrm{O}), 1658,1601,1526,1458,1380,1329,1146,1102,1067,1029 \mathrm{~cm}^{-1} . \mathrm{HRMS}(\mathrm{ESI}, \mathrm{m} / z):[\mathrm{M}-$ $\mathrm{H}^{-}$calcd 603.0136 for $\mathrm{C}_{24} \mathrm{H}_{22} \mathrm{Cl}_{2} \mathrm{O}_{12} \mathrm{~S}$ found 603.0137 .

3-( $\beta$-D-Glucopyranosyl-1-thio)-2,5,8-trimethoxynaphthalene-1,4-dione (14a). Quinone 10a $141 \mathrm{mg}$ (0.50 $\mathrm{mM})$ was dissolved in acetone $(10 \mathrm{~mL})$ and $\mathrm{MeOH}(10 \mathrm{~mL})$, thioglucose sodium salt $6110 \mathrm{mg}(0.50 \mathrm{mM})$ was added. The resulting mixture was stirred during $0.6 \mathrm{~h}$ at room temperature until the consumption of thioglucose 6 and conversion of the starting quinone 10a in the new orange compound with $R_{f} 0.45$ (B). Inorganic salts were filtered off, the precipitate was washed with acetone. The combined filtrate was evaporated in vacuum, the residue was subjected to preparative TLC (system B) and yielded 3-( $\beta$-Dglucopyranosyl-1-thio)-2,5,8-trimethoxynaphthalene-1,4-dione (14a); yield $177 \mathrm{mg}$ (80\%), brown solid, mp 129-131 ${ }^{\circ} \mathrm{C} .{ }^{1} \mathrm{H}$ NMR (500 MHz, DMSO- $\left.d_{6}\right): \delta 3.02\left(\mathrm{~m}, 1 \mathrm{H}, \mathrm{H}-5^{\prime}\right), 3.07\left(\mathrm{~m}, 1 \mathrm{H}, \mathrm{H}-2^{\prime}\right), 3.16\left(\mathrm{~m}, 1 \mathrm{H}, \mathrm{H}-4^{\prime}\right), 3.20(\mathrm{~m}$, $\left.1 \mathrm{H}, \mathrm{H}-3^{\prime}\right), 3.42\left(\mathrm{~m}, 2 \mathrm{H}, 2 \times \mathrm{H}^{\prime} 6^{\prime}\right), 3.81$ (s, 3H, ArOMe), 3.85 (s, 3H, ArOMe), 3.95 (s, 3H, ArOMe), $4.27(\mathrm{~m}, 1 \mathrm{H}$, $\left.\mathrm{C}^{6^{\prime}} \mathrm{H}_{2} \mathrm{O} \underline{\mathrm{H}}\right), 4.88\left(\mathrm{~d}, 1 \mathrm{H}, J 4.5 \mathrm{~Hz}, \mathrm{C}^{4} \mathrm{HO} \underline{\mathrm{H}}\right), 5.08\left(\mathrm{~m}, 1 \mathrm{H}, \mathrm{C}^{3} \mathrm{HO} \underline{\mathrm{H}}\right), 5.18\left(\mathrm{~d}, 1 \mathrm{H}, J 9.6 \mathrm{~Hz}, \mathrm{H}-1^{\prime}\right), 5.43(\mathrm{~d}, 1 \mathrm{H}, J 5.6 \mathrm{~Hz}$, $\left.\mathrm{C}^{2} \mathrm{HO} \underline{\mathrm{H}}\right), 7.45$ (d, $\left.1 \mathrm{H}, J 9.5 \mathrm{~Hz}, \mathrm{ArH}\right), 7.48(\mathrm{~d}, 1 \mathrm{H}, J 9.5 \mathrm{~Hz}, \mathrm{ArH}) .{ }^{13} \mathrm{C}$ NMR (DMSO-d, $\left.125 \mathrm{MHz}\right): \delta 56.6(\mathrm{MeO})$, 56.8 (MeO), 60.2 (MeO), 60.4 (C-6'), 69.6 (C-4'), 74.3 (C-2'), 78.2 (C-3'), 81.1 (C-5'), 82.0 (C-1'), 119.7, 120.3, 121.0, 121.5, 130.2, 152.2, 152.9, 156.2, $177.1(\mathrm{C}=\mathrm{O}), 181.3(\mathrm{C}=\mathrm{O}) . \mathrm{IR}(\mathrm{KBr}): 3444(\mathrm{OH}), 2938,1645(\mathrm{C}=\mathrm{O})$, 
1595, 1565, 1478, 1434, 1410, 1340, 1274, 1206, 1053, 1017, $917 \mathrm{~cm}^{-1}$. MS (ESI): m/z 465 [M+Na]. HRMS (ESI): calcd for $\mathrm{C}_{19} \mathrm{H}_{22} \mathrm{NaO}_{10} \mathrm{~S} 465.0826$, found 465.0821 .

6,7-Dichloro-3-( $\beta$-D-glucopyranosyl-1-thio)-2,5,8-trimethoxynaphthalene-1,4-dione (14b). Quinone $10 \mathrm{~b} 176$ $\mathrm{mg}(0.50 \mathrm{mM})$ was dissolved in acetone $(10 \mathrm{~mL})$ and $\mathrm{MeOH}(10 \mathrm{~mL})$ and thioglucose sodium salt $6110 \mathrm{mg}$ $(0.50 \mathrm{mM})$ was added. The resulting mixture was stirred during $1.0 \mathrm{~h}$ at room temperature until the consumption of thioglucose 6 and formation of two new orange compounds with $R_{f} 0,46(\mathrm{~B})$ and $R_{f} 0.49$ (B). The reaction mixture was evaporated under reduced pressure. The residue was subjected preparative TLC (system B) and led to tetracyclic quinone 12b, $R_{f} 0.49$ (B) $17 \mathrm{mg}$ (7\%), and 6,7-dichloro-3-( $\beta$-D-glucopyranosyl1-thio)-2,5,8-trimethoxynaphthalene-1,4-dione (14b); yield $189 \mathrm{mg}$ (74\%), brown solid, $\left.R_{f} 0.46 \mathrm{~B}\right), \mathrm{mp} 103-$ $105^{\circ} \mathrm{C} .{ }^{1} \mathrm{H}$ NMR (500 MHz, DMSO- $\left.d_{6}\right): \delta 3.03\left(\mathrm{~m}, 1 \mathrm{H}, \mathrm{H}-5^{\prime}\right), 3.09\left(\mathrm{~m}, 2 \mathrm{H}, \mathrm{H}-2^{\prime}, \mathrm{H}-4^{\prime}\right), 3.21\left(\mathrm{~m}, 1 \mathrm{H}, \mathrm{H}-3^{\prime}\right), 3.37(\mathrm{~m}$, $\left.1 \mathrm{H}, \mathrm{H}-6^{\prime}\right), 3.49$ (m, 1H, H-6'), 3.84 (s, 3H, OMe), 3.87 (s, 3H, OMe), 4.02 (s, 3H, OMe), $4.30\left(\mathrm{~m}, 1 \mathrm{H}, \mathrm{C}^{6} \mathrm{H}_{2} \mathrm{O} \underline{\mathrm{H}}\right)$, $4.92\left(\mathrm{~d}, 1 \mathrm{H}, J 5.2 \mathrm{~Hz}, \mathrm{C}^{4} \mathrm{HO} \underline{\mathrm{H}}\right), 5.11\left(\mathrm{~m}, 1 \mathrm{H}, \mathrm{C}^{3} \mathrm{HO} \underline{\mathrm{H}}\right), 5.21\left(\mathrm{~d}, 1 \mathrm{H}, J 9.8 \mathrm{~Hz}, \mathrm{H}-1^{\prime}\right), 5.48\left(\mathrm{~d}, 1 \mathrm{H}, J 6.4 \mathrm{~Hz}, \mathrm{C}^{2} \mathrm{HO} \underline{\mathrm{H}}\right)$. ${ }^{13} \mathrm{C} \mathrm{NMR}\left(\mathrm{DMSO}-d_{6}, 125 \mathrm{MHz}\right): \delta 60.6(\mathrm{MeO}), 60.7$ (C-6'), $61.8(\mathrm{MeO}), 62.1(\mathrm{MeO}), 69.8\left(\mathrm{C}-4^{\prime}\right), 74.4\left(\mathrm{C}-2^{\prime}\right), 78.1$ (C-3'), 81.3 (C-5'), 82.2 (C-1'), 124.6, 125.6, 130.2, 134.2, 134.3, 151.8, 152.0, 157.0, 175.9 (C=O), 179.9 (C=0). IR (KBr): $3430(\mathrm{OH}), 2939,1715,1662$ (C=O), 1583, 1546, 1459, 1381, 1326, 1273, 1180, 1117, $1026 \mathrm{~cm}^{-1} . \mathrm{MS}$ (ESI): $m / z 533$ [M+Na]. HRMS (ESI): calcd for $\mathrm{C}_{19} \mathrm{H}_{20} \mathrm{Cl}_{2} \mathrm{NaO}_{10} \mathrm{~S} 533.0046$, found 533.0046 .

2-Chloro-3-( $\beta$-D-glucopyranosyl-1-thio)-5,8-dihydroxynaphthalene-1,4-dione $\quad(15 \mathrm{a})$ and 2,3-di( $\beta$-Dglucopyranosyl-1-thio)-5,8-dihydroxynaphthalene-1,4-dione (15b). 2,3-Dichloroquinone 7a $130 \mathrm{mg}$ (0.50 $\mathrm{mM})$ was dissolved in acetone $(10 \mathrm{~mL})$ and $\mathrm{MeOH}(10 \mathrm{~mL})$ and thioglucose sodium salt $6194 \mathrm{mg}(0.91 \mathrm{mM})$ was added. The resulting mixture was stirred during $1.0 \mathrm{~h}$ at room temperature until the consumption of thioglucose 6 and formation of two new red compounds with $R_{f} 0.55$ (B) and $R_{f} 0.20$ (B). The reaction mixture was evaporated under reduced pressure. The residue was subjected preparative TLC (system B) and yielded 2chloro-3-( $\beta$-D-glucopyranosyl-1-thio)-5,8-dihydroxynaphthalene-1,4-dione (15a); yield $44 \mathrm{mg}$ (21 \%), dark red solid, mp 154-157 ${ }^{\circ} \mathrm{C} .{ }^{1} \mathrm{H}$ NMR (700 MHz, DMSO-d $\left.d_{6}\right): \delta 3.10\left(\mathrm{t}, 1 \mathrm{H}, \mathrm{H}-3^{\prime}\right), 3.11\left(\mathrm{~m}, 1 \mathrm{H}, \mathrm{H}-5^{\prime}\right), 3.15$ (t, $1 \mathrm{H}, J 9.2 \mathrm{~Hz}$, $\mathrm{H}^{-5}$ '), 3.23 (t, 1H, J $\left.8.3 \mathrm{~Hz}, \mathrm{H}-4^{\prime}\right), 3.31$ (dd, 1H, J 5.7, $12 \mathrm{~Hz}, \mathrm{H}-6$ ), 3.46 (dd, 1H, J 1.9, $12 \mathrm{~Hz}, \mathrm{H}-6$ '), 4.25 (br.s, $2 \mathrm{H}$, $2 \times \mathrm{OH}), 4.94$ (br.s, $2 \mathrm{H}, 2 \times \mathrm{OH}), 5.55\left(\mathrm{~d}, 1 \mathrm{H}, J 9.7 \mathrm{~Hz}, \mathrm{H}-1^{\prime}\right), 7.39$ (s, 2H, $\left.2 \times \mathrm{ArH}\right), 11.84(\mathrm{~s}, 1 \mathrm{H}, \alpha-\mathrm{OH}), 12.01(\mathrm{~s}$, $1 \mathrm{H}, \alpha-\mathrm{OH}) .{ }^{13} \mathrm{C}$ NMR (DMSO- $d_{6}, 176 \mathrm{MHz}$ ): $\delta 60.7$ (C-6'), 70.0 (C-4'), 74.9 (C-2'), 78.2 (C-3'), 81.7 (C-5'), 83.7 (C$\left.1^{\prime}\right), 111.4,112.2,129.2,129.4,140.6,147.9,156.9,157.1,177.9$ (C=O), 181.9 (C=O). IR (KBr): 3368 (OH), 2919, 1711,1617 (C=O), 1574, 1535, 1450, 1403, 1358, 1309, 1265, 1226, 1201, 1088, 1050, 988, 880, $780 \mathrm{~cm}^{-1}$. MS (ESI): $m / z 441$ [M+Na]. HRMS (ESI): calcd for $\mathrm{C}_{16} \mathrm{H}_{15} \mathrm{ClNaO}_{9} \mathrm{~S} 441.0018$, found 441.0017 and 2,3-di( $\beta-\mathrm{D}-$ glucopyranosyl-1-thio)-5,8-dihydroxynaphthalene-1,4-dione (15b). Yield $199 \mathrm{mg}$ (69\%), red solid. mp 179-181 ${ }^{\circ} \mathrm{C} .{ }^{1} \mathrm{H}$ NMR $\left(500 \mathrm{MHz}, \mathrm{DMSO}-d_{6}\right): \delta 3.09\left(\mathrm{~m}, 4 \mathrm{H}, 2 \times \mathrm{H}-4^{\prime}, 2 \times \mathrm{H}-5^{\prime}\right), 3.16\left(\mathrm{dd}, 2 \mathrm{H}, J 8.5,9.7 \mathrm{~Hz}, 2 \times \mathrm{H}-2^{\prime}\right), 3.20$ (dd, $\left.2 \mathrm{H}, J 8.2,8.5 \mathrm{~Hz}, 2 \times \mathrm{H}_{-} 3^{\prime}\right), 3.32\left(\mathrm{dd}, 2 \mathrm{H}, J 5.0,12.0 \mathrm{~Hz}, 2 \times \mathrm{H}-6^{\prime}\right), 3.48\left(\mathrm{~d}, 2 \mathrm{H}, J 12.0 \mathrm{~Hz}, 2 \times \mathrm{H}^{\prime} 6^{\prime}\right), 4.28$ (br.s, $2 \mathrm{H}, 2 \times \mathrm{C}^{6^{\prime}} \mathrm{H}_{2} \mathrm{OH}$ ), 4.95 (br.s, $\left.4 \mathrm{H}, 2 \times \mathrm{C}^{3} \mathrm{HO} \underline{\mathrm{H}}, 2 \times \mathrm{C}^{4^{\prime}} \mathrm{HO} \underline{\mathrm{H}}\right), 5.40$ (d, $\left.2 \mathrm{H}, J 8.4 \mathrm{~Hz}, 2 \times \mathrm{H}-1^{\prime}\right), 5.52$ (br.s, $2 \mathrm{H}, 2 \times$ $\left.\mathrm{C}^{2} \mathrm{HO} \underline{\mathrm{H}}\right), 7.33$ (s, 2H, H-6, H-7), 12.06 (s, 2H, $\left.2 \times \alpha-\mathrm{OH}\right) .{ }^{13} \mathrm{C}$ NMR (DMSO-d, $\left.125 \mathrm{MHz}\right): \delta 60.8$ (C-6'), 70.0 (C-4'), 75.0 (C-2'), 78.2 (C-3'), 81.6 (C-5'), 84.0 (C-1'), 112.1, 128.6, 147.3, 156.4, $181.9(\mathrm{C}=\mathrm{O}) . \mathrm{IR}(\mathrm{KBr}): 3421(\mathrm{OH})$, 2922, 2360, 1615 (C=O), 1456, 1403, 1485, 1361, 1235, 1200, 1164, 1075, $1039 \mathrm{~cm}^{-1} . \mathrm{MS}$ (ESI): $\mathrm{m} / \mathrm{z} 601$ [M+Na]. HRMS (ESI): calcd for $\mathrm{C}_{22} \mathrm{H}_{26} \mathrm{NaO}_{14} \mathrm{~S}_{2} 601.0656$, found 601.0658 .

\section{2,3-Di( $\beta$-D-glucopyranosyl-1-thio)-5,8-dihydroxy-6,7-dimethylnaphthalene-1,4-dione}

(15c).

$2,3-$

Dichloroquinone $7 \mathrm{~b} 144 \mathrm{mg}(0.50 \mathrm{mM})$ was dissolved in acetone $(10 \mathrm{~mL})$ and $\mathrm{MeOH}(10 \mathrm{~mL})$ and thioglucose sodium salt $6275 \mathrm{mg}(1.26 \mathrm{mM})$ was added. The resulting mixture was stirred during $18 \mathrm{~h}$ at room temperature until the consumption of quinone $\mathbf{7 b}$ and formation of the new red compound with $R_{f} 0.23$ (B). The reaction mixture was evaporated under reduced pressure. The residue was subjected preparative TLC (system B) and yielded 2,3-di( $\beta$-D-glucopyranosyl-1-thio)-5,8-dihydroxy-6,7-dimethylnaphthalene-1,4-dione (15c); yield $288 \mathrm{mg}$ (95\%), red solid, mp 198-200 ${ }^{\circ} \mathrm{C} .{ }^{1} \mathrm{H} \mathrm{NMR}\left(700 \mathrm{MHz}, \mathrm{DMSO}-d_{6}\right): \delta 2.22\left(\mathrm{~s}, 6 \mathrm{H}, 2 \times \mathrm{ArCH}_{3}\right)$, 
3.05 (ddd, $2 \mathrm{H}, J 1.8,5.6,9.6 \mathrm{~Hz}, 2 \times \mathrm{H}^{-5}$ '), 3.10 (dd, 2H, J 8.8, 9.5 Hz, $2 \times \mathrm{H}-4$ '), 3.15 (dd, 2H, J 8.8, $9.6 \mathrm{~Hz}, 2 \times \mathrm{H}-$ $\left.2^{\prime}\right), 3.21$ (dd, $\left.2 \mathrm{H}, J 8.6 \mathrm{~Hz}, 2 \times \mathrm{H}-3^{\prime}\right), 3.33$ (dd, 2H, J 5.6, $12.0 \mathrm{~Hz}, 2 \times \mathrm{H}-6$ '), 3.45 (dd, 2H, J 1.8, $\left.12.0 \mathrm{~Hz}, 2 \times \mathrm{H}^{\prime} 6^{\prime}\right)$, 4.30 (br.s, $2 \mathrm{H}, 2 \times \mathrm{C}^{6^{\prime}} \mathrm{H}_{2} \mathrm{O} \underline{\mathrm{H}}$ ), 4.99 (br.s, $\left.2 \mathrm{H}, 2 \times \mathrm{C}^{4} \mathrm{HO} \underline{\mathrm{H}}\right), 5.15$ (br.s, $\left.2 \mathrm{H}, 2 \times \mathrm{C}^{3} \mathrm{HO} \underline{\mathrm{H}}\right), 5.41$ (d, $2 \mathrm{H}, J 9.6 \mathrm{~Hz}, 2 \times \mathrm{H}-$ $\left.1^{\prime}\right), 5.51$ (br.s, $\left.\left.2 \mathrm{H}, 2 \times \mathrm{C}^{2} \mathrm{HO} \underline{\mathrm{H}}\right),\right), 13.09$ (s, 2H, $\left.2 \times \alpha-\mathrm{OH}\right) .{ }^{13} \mathrm{C}$ NMR (DMSO-d, $\left.176 \mathrm{MHz}\right): \delta$ 12.28, $\left(\mathrm{ArCH}_{3}\right), 60.7$ (C-6'), 69.9 (C-4'), 75.0 (C-2'), 78.2 (C-3'), 81.5 (C-5'), 84.0 (C-1'), 109.0, 138.1, 145.8, 160.6, 178.2 (C=O). IR (KBr): 3409 (OH), 2890, 1600 (C=O), 1447, 1400, 1360, 1268, 1213, 1096, 1076, 1050, 878, $809 \mathrm{~cm}^{-1}$. MS (ESI): $\mathrm{m} / z 629$ [M+Na]. HRMS (ESI): calcd for $\mathrm{C}_{24} \mathrm{H}_{30} \mathrm{NaO}_{14} \mathrm{~S}_{2} 629.0969$, found 629.0956.

$(2 R, 3 R, 4 S, 4 a R, 12 a S)-2-H y d r o x y m e t h y l-3,4,7,10$-tetrahydroxy-3,4,4a,12a-tetrahydro-2H-naphtho[2,3-b]pyrano[2,3-e][1,4]-oxathiine-6,11-dione (16a). Dithioglucoside 15b $87 \mathrm{mg}(0.15 \mathrm{mM})$ was dissolved in $\mathrm{MeOH}$ $(30 \mathrm{~mL})$ and $0.5 \mathrm{~N}$ solution of $\mathrm{MeONa} / \mathrm{MeOH}(1.4 \mathrm{~mL}, 0.70 \mathrm{mM})$ was added. The reaction mixture changed color from red to dark blue and the dark blue precipitate of bisthiogluside 15b sodium salt was formed. The reaction mixture was stirred at room temperature during $21 \mathrm{~h}$, until consumption of bisthiogluside 15b, acidified by dropwise addition of conc. $\mathrm{HCl}$ and was evaporated under reduced pressure. The residue was subjected preparative TLC (system C). The main red band with $R_{f} 0.43$ was eluted from $\mathrm{SiO}_{2}$ with acetone. The acetone eluate was dissolved by equal volume of $\mathrm{MeOH}$ and the solution was gently evaporated on reduced pressure and led $(2 R, 3 R, 4 S, 4 a R, 12 \mathrm{aS})$-2-hydroxymethyl-3,4,7,10-tetrahydroxy-3,4,4a,12a-tetrahydro- $2 \mathrm{H}$ naphtho[2,3-b]pyrano[2,3-e][1,4]-oxathiine-6,11-dione (16a); yield $10 \mathrm{mg}(17 \%)$, red solid, $\mathrm{mp} 312-315^{\circ} \mathrm{C} .{ }^{1} \mathrm{H}$ NMR (700 MHz, DMSO- $\left.d_{6}\right): \delta 3.33(\mathrm{~m}, 1 \mathrm{H}, \mathrm{H}-3), 3.50(\mathrm{~m}, 1 \mathrm{H}, \mathrm{H}-2), 3.51(\mathrm{~m}, 1 \mathrm{H}, \mathrm{H}-13), 3.61(\mathrm{~m}, 2 \mathrm{H}, \mathrm{H} 4, \mathrm{H}-4 \mathrm{a})$, $3.75(\mathrm{~m}, 1 \mathrm{H}, \mathrm{H}-13), 4.74\left(\mathrm{~m}, 1 \mathrm{H}, \mathrm{C}^{13} \mathrm{H}_{2} \mathrm{O} \underline{\mathrm{H}}\right), 5.03(\mathrm{~m}, 1 \mathrm{H}, \mathrm{H}-12 \mathrm{a}), 5.43\left(\mathrm{~d}, 1 \mathrm{H}, J 5.8 \mathrm{~Hz}, \mathrm{C}^{3} \mathrm{HO} \underline{\mathrm{H}}\right), 5.70(\mathrm{~m}, 1 \mathrm{H}$, $\left.\mathrm{C}^{4} \mathrm{HO} \underline{\mathrm{H}}\right), 7.34(\mathrm{~d}, 1 \mathrm{H}, J 9.3 \mathrm{~Hz}, \mathrm{ArH}), 7.36(\mathrm{~d}, 1 \mathrm{H}, J 9.3 \mathrm{~Hz}, \mathrm{ArH}), 11.88(\mathrm{~s}, 1 \mathrm{H}, \alpha-\mathrm{OH}), 12.15 .(\mathrm{s}, 1 \mathrm{H}, \alpha-\mathrm{OH}) .{ }^{13} \mathrm{C}$ NMR (DMSO- $d_{6}, 176 \mathrm{MHz}$ ): $\delta 60.7$ (C-13), 70.4 (C-3), 73.4 (C-12a), 73.8 (C-4), 79.3 (C-4a), 82.3 (C-2), 110.4, 110.5, 124.0, 129.3 (2 × ArH), 150.6, 155.8, 156.7, $178.9(\mathrm{C}=\mathrm{O}), 184.3$ (C=O). IR (KBr): $3402(\mathrm{OH}), 2923,1601$ (C=O), 1579, 1448, 1413, 1241, 1223, 1182, 1142, 1077, $979 \mathrm{~cm}^{-1}$. MS (ESI): $\mathrm{m} / \mathrm{z} 405$ [M+Na]. HRMS (ESI): calcd for $\mathrm{C}_{16} \mathrm{H}_{14} \mathrm{NaO}_{9} \mathrm{~S} 405.0251$, found 405.0255 .

\section{(2R,3R,4S,4aR,12aS)-2-Hydroxymethyl-3,4,7,10-tetrahydroxy-8,9-dimethyl-3,4,4a,12a-tetrahydro-2H-}

naphtho[2,3-b]pyrano[2,3-e][1,4]-oxathiine-6,11-dione (16b). Dithioglucoside 15c $91 \mathrm{mg}(0.15 \mathrm{mM})$ was dissolved in $\mathrm{MeOH}(30 \mathrm{~mL})$ and $0.5 \mathrm{~N}$ solution of $\mathrm{MeONa} / \mathrm{MeOH}(1.4 \mathrm{~mL}, 0.70 \mathrm{mM})$ was added with formation of dark blue suspension of bisthioglucoside $15 \mathrm{c}$ sodium salt. The reaction mixture changed color from red to dark blue and the dark blue solution was formed. The reaction mixture was stirred at room temperature during $6 \mathrm{~h}$, until consumption of bisthioglucoside 15c, acidified by dropwise addition of conc. $\mathrm{HCl}$ and was evaporated under reduced pressure. The residue was subjected preparative TLC (system C). The main red band with $R_{f} 0.47$ was eluted from $\mathrm{SiO}_{2}$ with acetone. The acetone eluate was dissolved by equal volume of $\mathrm{MeOH}$ and the solution was gently evaporated on reduced pressure and led the sample $(2 R, 3 R, 4 S, 4 \mathrm{a} R, 12 \mathrm{a} S)-2-$ hydroxymethyl-3,4,7,10-tetrahydroxy-8,9-dimethyl-3,4,4a,12a-tetrahydro-2H-naphtho[2,3-b]pyrano[2,3-

e][1,4]-oxathiine-6,11-dione (16b); yield $53 \mathrm{mg}$ (85\%), red solid, mp 315-318 ${ }^{\circ} \mathrm{C} .{ }^{1} \mathrm{H}$ NMR (500 MHz, DMSO- $\left.d_{6}\right)$ : $\delta 2.18$ (s, 3H, ArMe), 2.19 (s, 3H, ArMe), 3.32 (m, 1H, H-3), 3.50 (m, 2H, H-2, H-13), 3.60 (m, 2H, H-4, H-4a), $3.75(\mathrm{~m}, 1 \mathrm{H}, \mathrm{H}-13), 4.81\left(\mathrm{~m}, 1 \mathrm{H}, \mathrm{C}^{13} \mathrm{H}_{2} \mathrm{OH}\right), 5.01(\mathrm{~m}, 1 \mathrm{H}, \mathrm{H}-12 \mathrm{a}), 5.49\left(\mathrm{~d}, 1 \mathrm{H}, J 5.9 \mathrm{~Hz}, \mathrm{C}^{3} \mathrm{HOH}\right), 5.76(\mathrm{~m}, 1 \mathrm{H}$, $\left.\mathrm{C}^{4} \mathrm{HO} \underline{\mathrm{H}}\right), 12.64(\mathrm{~s}, 1 \mathrm{H}, \alpha-\mathrm{OH}), 12.94 .(\mathrm{s}, 1 \mathrm{H}, \alpha-\mathrm{OH}) .{ }^{13} \mathrm{C} N M R\left(\mathrm{DMSO}-d_{6}, 125 \mathrm{MHz}\right): \delta 12.1(\mathrm{Me}), 12.2(\mathrm{Me}), 60.7$ (C-13), 70.4 (C-3), 73.4 (C-12a), 73.9 (C-4), 79.3 (C-4a), 82.3 (C-2), 117.3, 107.4, 123.5, 137.8, 137.9, 150.4, 156.3, 157.3, 177.7 (C=O), 183.0 (C=O). IR (KBr): 3416 (OH), 2925, 1595 (C=O), 1570, 1448, 1387, 1307, 1266, 1181, 1089, 1076, 1038, 979, $809 \mathrm{~cm}^{-1}$. MS (ESI): $\mathrm{m} / z 433$ [M+Na]. HRMS (ESI): calcd for $\mathrm{C}_{18} \mathrm{H}_{18} \mathrm{NaO}_{9} \mathrm{~S}$ 433.0564, found 433.0554 .

2-( $\beta$-D-Glucopyranosyl-1-thio)-5,8-dihydroxy-3-methoxynaphthalene-1,4-dione (17a). Quinone 8a $113 \mathrm{mg}$ $(0.40 \mathrm{mM})$ was dissolved in acetone $(10 \mathrm{~mL})$ and $\mathrm{MeOH}(10 \mathrm{~mL})$, thioglucose sodium salt $6110 \mathrm{mg}(0.50 \mathrm{mM})$ was added. The resulting mixture was stirred during $1.5 \mathrm{~h}$ at room temperature until the consumption of 
thioglucose 6 and conversion of the starting quinone $8 \mathrm{a}$ in two new red compounds with $R_{f} 0.45$ (B) and $R_{f}$ 0.20 (B). The resulting mixture was evaporated in vacuum and residue was subjected preparative TLC and gave 2-( $\beta$-D-glucopyranosyl-1-thio)-5,8-dihydroxy-3-methoxynaphthalene-1,4-dione (17a); yield $132 \mathrm{mg}(80 \%)$, red solid, $R_{f} 0.45$ (B), mp 192-194 ${ }^{\circ} \mathrm{C} .{ }^{1} \mathrm{H}$ NMR (500 MHz, DMSO- $\left.d_{6}\right): \delta 3.09\left(\mathrm{~m}, 3 \mathrm{H}, \mathrm{H}-2^{\prime}, \mathrm{H}-4^{\prime}, \mathrm{H}-5^{\prime}\right), 3.22(\mathrm{~m}, 1 \mathrm{H}, \mathrm{H}-$ $\left.3^{\prime}\right), 3.35\left(\mathrm{~m}, 1 \mathrm{H}, \mathrm{H}-6^{\prime}\right), 3.56\left(\mathrm{~m}, 1 \mathrm{H}, \mathrm{H}^{\prime} 6^{\prime}\right), 4.15(\mathrm{~s}, 1 \mathrm{H}, \mathrm{OMe}), 4.37\left(\mathrm{t}, 1 \mathrm{H}, J 5.9 \mathrm{~Hz}, \mathrm{C}^{6} \mathrm{H}_{2} \mathrm{O} \underline{\mathrm{H}}\right), 4.95(\mathrm{~d}, 1 \mathrm{H}, J 5.4 \mathrm{~Hz}$, $\left.\mathrm{C}^{4^{\prime}} \mathrm{HO} \underline{\mathrm{H}}\right), 5.11\left(\mathrm{~d}, 1 \mathrm{H}, J 5.0 \mathrm{~Hz}, \mathrm{C}^{3^{\prime}} \mathrm{HO} \underline{\mathrm{H}}\right), 5.27\left(\mathrm{~d}, 1 \mathrm{H}, J 10.0 \mathrm{~Hz}, \mathrm{H}-1^{\prime}\right), 5.48\left(\mathrm{~m}, 1 \mathrm{H}, J 6.5 \mathrm{~Hz}, \mathrm{C}^{2} \mathrm{HOH}\right), 7.35$ (s, $2 \mathrm{H}, 2$ $\times \mathrm{Ar}-\mathrm{H}), 12.02(\mathrm{~s}, 1 \mathrm{H}, \alpha-\mathrm{OH}), 12.21(\mathrm{~s}, 1 \mathrm{H}, \alpha-\mathrm{OH}) .{ }^{13} \mathrm{C}$ NMR (DMSO-d, $\left.125 \mathrm{MHz}\right): \delta 61.0$ (C-6'), 61.5 (MeO), 70.0 (C-4'), 74.5 (C-2'), 78.1 (C-3'), 81.4 (C-5'), 82.7 (C-1'), 111.3, 111.7, 128.8, 129.2, 131.5, 156.0, 156.5, 158.4, 181.5 (C=O), 186.1 (C=O). IR ( $\mathrm{KBr}): 3420(\mathrm{OH}), 2948,2886,1608$ (C=O), 1557, 1455, 1407, 1263, 1192, 1135, 1263, 1192, 1135, 1079, 1063, 1035, $980 \mathrm{~cm}^{-1}$. MS (ESI): $\mathrm{m} / z 465$ [M+Na]. HRMS (ESI): calcd for $\mathrm{C}_{17} \mathrm{H}_{18} \mathrm{NaO}_{10} \mathrm{~S}$ 437.0513, found 437.0512 and known dithioglucoside $15 \mathrm{a} 35 \mathrm{mg}(15 \%)$.

2-( $\beta$-D-Glucopyranosyl-1-thio)-5,8-dihydroxy-3-methoxy-6,7-dimethylnaphthalene-1,4-dione (17b). Quinone 8b $99 \mathrm{mg}(0.35 \mathrm{mM})$ was dissolved in acetone $(10 \mathrm{~mL})$ and $\mathrm{MeOH}(10 \mathrm{~mL})$, thioglucose sodium salt $6110 \mathrm{mg}$ (0.45 $\mathrm{mM}$ ) was added. The resulting mixture was stirred during $1.5 \mathrm{~h}$ at room temperature until the consumption of thioglucose $\mathbf{6}$ was observed. The starting quinone $\mathbf{8 b}$ was converted in three new red compounds with $R_{f} 0.47$ (B), $R_{f} 0.40$ (B) and $R_{f} 0.10$ (B). The reaction mixture was evaporated in vacuum and residue was subjected preparative TLC and gave tetracyclic quinone $16 \mathrm{~b}, R_{f} 0.47(\mathrm{~B})$, yield $8 \mathrm{mg}(5.5 \%)$, 2-( $\beta-\mathrm{D}-$ glucopyranosyl-1-thio)-5,8-dihydroxy-3-methoxy-6,7-dimethylnaphthalene-1,4-dione (17b), $R_{f} 0.40$ (B), yield $130 \mathrm{mg}(84 \%)$, red solid, mp 195-196 ${ }^{\circ} \mathrm{C} .{ }^{1} \mathrm{H}$ NMR (500 MHz, DMSO-d $\left.{ }_{6}\right): \delta 2.21(\mathrm{~s}, 6 \mathrm{H}, 2 \times 0 M e), 3.09(\mathrm{~m}, 3 \mathrm{H}, \mathrm{H}-$ 2', H-4', H-5'), 3.23 (m, 1H, H-3'), 3.35 (dd, 1H, J 5.5, $\left.12.0 \mathrm{~Hz}, \mathrm{H}-6^{\prime}\right), 3.55$ (dd, 1H, J 1.7, $\left.12.0 \mathrm{~Hz}, \mathrm{H}-6^{\prime}\right), 4.13$ (s, $1 \mathrm{H}, \mathrm{OMe}$ ), 4.35 (br.s, $1 \mathrm{H}, \mathrm{C}^{6} \mathrm{H}_{2} \mathrm{O} \underline{\mathrm{H}}$ ), 4.97 (br.s, 2H, $\left.\mathrm{C}^{3} \mathrm{HO} \underline{\mathrm{H}}, \mathrm{C}^{4} \mathrm{HO} \underline{\mathrm{H}}\right), 5.27$ (d, $\left.1 \mathrm{H}, J 9.8 \mathrm{~Hz}, \mathrm{H}-1^{\prime}\right), 5.45$ (br.s, $1 \mathrm{H}$, $\left.\mathrm{C}^{2} \mathrm{HO} \underline{\mathrm{H}}\right), 12.90$ (s, $\left.1 \mathrm{H}, \alpha-\mathrm{OH}\right), 13.13(\mathrm{~s}, 1 \mathrm{H}, \alpha-\mathrm{OH}) .{ }^{13} \mathrm{C} N M R$ (DMSO-d, $\left.125 \mathrm{MHz}\right): \delta 12.1(\mathrm{Me}), 12.3(\mathrm{Me}), 61.0$ (C-6'), 61.4 (MeO), 70.0 (C-4'), 74.5 (C-2'), 78.1 (C-3'), 81.4 (C-5'), 82.9 (C-1'), 108.0, 109.0, 130.7, 138.0, 138.6, 158.0, 159.8, 160.4, 177.3 (C=O), 182.1 (C=O). IR (KBr): 3408 (OH), 2924, 1737, 1601 (C=O), 1555, 1444, 1387, $1260,1181,1141,1098,1075,1034,808 \mathrm{~cm}^{-1}$. MS (ESI): $\mathrm{m} / z 465$ [M+Na]. HRMS (ESI): calcd for $\mathrm{C}_{19} \mathrm{H}_{22} \mathrm{NaO}_{10} \mathrm{~S}$ 465.0826, found 465.0821, and dithioglucoside 15c, yield $11 \mathrm{mg}(5 \%)$.

Preparation of tetracyclic quinones $16 \mathbf{a}, \mathbf{b}$ from 3-methoxy-2-thioglucosides $17 \mathbf{a}, \mathbf{b}$. Thioglucoside $17 \mathbf{a}, \mathbf{b}(0.19$ $\mathrm{mM}$ ) was dissolved in $\mathrm{MeOH}(30 \mathrm{~mL})$ and $0.5 \mathrm{~N}$ solution of $\mathrm{MeONa} / \mathrm{MeOH}(1.5 \mathrm{~mL}, 0.75 \mathrm{mM})$ was added and the dark blue solution was formed. The reaction mixture was stirred at room temperature during $6 \mathrm{~h}$ for $17 \mathrm{a}$ and $2.5 \mathrm{~h}$ for $\mathbf{1 7 b}$ until conversion of starting quinones in tetracycles $\mathbf{1 6 a , b}$. The reaction mixture was acidified by dropwise addition of conc. $\mathrm{HCl}$ and was evaporated under reduced pressure. The residue was subjected preparative TLC (system C). The main red band was eluted from $\mathrm{SiO}_{2}$ with acetone, the eluate was diluted with equal volume of $\mathrm{MeOH}$ and gently evaporated on reduced pressure and led to 16a, $R_{f} 0.43$ (B), yield $65 \mathrm{mg}$ (90\%) and 16b, $R_{f} 0.47(\mathrm{~B})$, yield $71 \mathrm{mg}(91 \%)$.

\section{Acknowledgements}

The authors acknowledge Dr. V.P. Glazunov and Ms. O.P. Moiseenko for IR and MS measurements. The authors appreciate Dr. V.I. Kalinin and Dr. N.S Polonik for helpful discussion and correction of the manuscript. 


\section{Supplementary Material}

${ }^{1} \mathrm{H}$ and ${ }^{13} \mathrm{C}$ NMR spectra are provided for all new compounds: $4 \mathbf{a}, \mathbf{b} ; \mathbf{8 b} ; \mathbf{1 0 b} ; \mathbf{1 1 a , b} ; \mathbf{1 2 a}, \mathbf{b} ; \mathbf{1 3 a} \mathbf{b} \mathbf{b} ; \mathbf{1 4 a , b} ; \mathbf{1 5 a}, \mathbf{b}$; $16 a, b$ and $17 a, b$.

\section{References}

1. Thomson, R. H. Naturally occurring quinones IV; Blackie Academic \& Professional, an imprint of Chapman \& Hall: London, 1997.

2. Sanchez-Calvo, J. M.; Barbero, G. R.; Guerrera-Vasquez, G.; Duran, A. G.; Macıas, M.; Rodriguez-Iglesias, M. A.; Molinillo, J. M. G.; Macias, F. A. Med. Chem. Res. 2016, 25, 1274. http://dx.doi:10.1007/s00044-016-1550-x

3. Mishchenko, N. P, Fedoreev, S. A.; Bagirova, V. L. Pharm. Chem. J. 2003, 37, 48.

4. Asche, C. Mini Rev. Med. Chem. 2005, 5, 449. http://dx.doi:10.2174/1389557053765556

5. Wellington, K. W. RSC Advances 2015, 5, 20309. http://dx.doi:10.1039/C4RA13547D

6. Klotz, L.; Hou, X.; Jacob, C. Molecules 2014, 19, 14902. https://doi.org/10.3390/molecules190914902

7. Constantino, L.; Barlocco, D. Curr. Med. Chem. 2006, 13, 65. http://dx.doi:10.2174/092986706775197999

8. Polonik, S. G.; Prokof'eva, N. G.; Agafonova, I. G.; Uvarova, N. I. Pharm. Chem. J. 2003, 37, 397.

9. Su, Y.; Xie, J.; Wang, Y.; Hub, X.; Lin,X. Eur. J. Med. Chem. 2010, 45, 2713. http://dx.doi.org/10.1016/j.ejmech.2010.02.002

10. Pelageev, D. N.; Dyshlovoy. S. A.; Pokhilo, N. D.; Denisenko, V. A.; Borisova, K. L.; von Amsberg, G. K.; Bokemeyer, C.; Fedorov, S. N.; Honecker, F.; Anufriev, V. Ph. Eur. J. Med. Chem. 2014, 77, 139. https://dx.doi.org/10.1016/i.ejmech.2014.03.006

11. Lin, H.-Y.; Han, H.-W.; Bai, L.-F.; Qiu, H,-Y.; De-Zheng, Y.; Jin-Liang Qi, J.-L.; Wang, X.-M.; Gu, H.-W.; Yang, Y.-H. RSC. Adv. 2014, 4, 49796.

http://dx.doi:10.1039/C4RA08810G

12. Polonik, S. G.; Tolkach, A. M.; Uvarova, N. I. Russ. Chem. Bull. Int. Ed. 1996, 45, 459. http://dx.doi:10.1007/BF01433995

13. Polonik, S. G.; Denisenko, V. A. Russ. Chem. Bull. Int. Ed. 2009, 58, 1062. http://dx.doi:10.1007/s11172-009-0135-y

14. Fedorov, S. N.; Shubina, L. K.; Kuzmich, A. S.; Polonik, S. G. Open Glycoscience 2011, 4, 1.

15. Huot, R.; Brassard. P. Can. J. Chem. 1974, 54, 838. http://dx.doi.org/10.1139/

16. Anufriev, V. P.; Polonik, S. G.; Pokhilo, N. D.; Balanyova, N. N. Russ. Chem. Bull. Int. Ed. 2003, $52,2247$. https://doi.org/10.1023/B:RUCB.0000011886.03785.bd

17. Shestak, O. P.; Anufriev, V. P.; Novikov, V.L. Nat. Prod. Commun. 2014, 9, 953.

18. Sabutskii, Y. E.; Semenova, M. N.; Yurchenko, E. A.; Polonik, N. S.; Denisenko, V. A.; Dmitrenok, P. S.; Semenov, V. V.; Polonik, S. G. Nat. Prod. Commun. 2017, 12, (in press).

19. Matsuoka, M.; Hamano, K.; Kitao, T. Synthesis 1984, 953. 
http://dx.doi:10.1055/s-1984-31034 